\title{
On the Global Dissipative and Multipeakon Dissipative Behavior of the Two-Component Camassa-Holm System
}

\author{
Yujuan Wang, ${ }^{1}$ Yongduan Song, ${ }^{1}$ and Hamid Reza Karimi ${ }^{2}$ \\ ${ }^{1}$ School of Automation, Chongqing University, Chongqing 400044, China \\ ${ }^{2}$ Department of Engineering, Faculty of Engineering and Science, University of Agder, 4898 Grimstad, Norway
}

Correspondence should be addressed to Yongduan Song; ydsong@cqu.edu.cn

Received 14 February 2014; Revised 20 March 2014; Accepted 20 March 2014; Published 4 May 2014

Academic Editor: Bo Shen

Copyright (c) 2014 Yujuan Wang et al. This is an open access article distributed under the Creative Commons Attribution License, which permits unrestricted use, distribution, and reproduction in any medium, provided the original work is properly cited.

\begin{abstract}
The global dissipative and multipeakon dissipative behavior of the two-component Camassa-Holm shallow water system after wave breaking was studied in this paper. The underlying approach is based on a skillfully defined characteristic and a set of newly introduced variables which transform the original system into a Lagrangian semilinear system. It is the transformation, together with the associated properties, that allows for the continuity of the solution beyond collision time to be established, leading to a uniquely global dissipative solution, which constructs a semigroup, and the multipeakon dissipative solution.
\end{abstract}

\section{Introduction}

In view of the wide applications in fluid dynamics, nonlinear optics, biochemistry, microbiology, physics, and many other fields, the study of the dynamic behavior of shallow water wave represents an important subject of research [1-5]. The Camassa-Holm $(\mathrm{CH})$ equation [1] has been widely used to model the unidirectional propagation of shallow water waves over a flat bottom. The nonstandard properties of the $\mathrm{CH}$ equation set it apart from the classical soliton equations such as $\mathrm{KdV}$, the first two remarkable of which are that it has peaked solitons $[1,6]$ and is able to model wave breaking $[1,2]$. The presence of breaking waves means that the solution remains bounded while its slope becomes unbounded in finite time $[2,5]$. After wave breaking, the solutions of the $\mathrm{CH}$ equation become uniquely as either global conservative [7-9] or global dissipative solutions [10-12].

In this paper, we focus on the two-component CamassaHolm shallow water system [13-15]:

$$
\begin{gathered}
m_{t}+u m_{x}+2 u_{x} m+\sigma \rho \rho_{x}=0, \quad t>0, x \in R, \\
\rho_{t}+(u \rho)_{x}=0, \quad t>0, x \in R,
\end{gathered}
$$

with $m=\sigma_{1} u-u_{x x}, \sigma=1, \sigma_{1}=1$ ( or in the "short wave" limit, $\left.\sigma_{1}=0\right)$. Here $u(x, t)$ represents the horizontal velocity of the fluid and $\rho(x, t)$ is in connection with the free-surface elevation from equilibrium (or scalar density). This system appears originally in [16] and then derived by Constantin and Ivanov [13] in the context of shallow water theory. System (1) is an extension of the CamassaHolm $(\mathrm{CH})$ equation by combing its integrability property with compressibility or free-surface elevation dynamics in its shallow water interpretation [6, 17]; analogously it is formally integrable [13-15] in the sense that it can be written as a compatibility condition of two linear systems (Lax pair) with a spectral parameter $\zeta$ :

$$
\begin{gathered}
\Psi_{x x}=\left(-\sigma \zeta^{2} \rho^{2}+\zeta m+\frac{\sigma_{1}}{4}\right) \Psi, \\
\Psi_{t}=\left(\frac{1}{2} \zeta-u\right) \Psi_{x}+\frac{1}{2} \cdot u_{x} \Psi .
\end{gathered}
$$

The Cauchy problem for the two-component CamassaHolm (CH2) system has been studied extensively [18-22]. It has been shown that the $\mathrm{CH} 2$ system is locally well-posed with initial data $\left(u_{0}, \rho_{0}\right) \in H^{s} \times H^{s-1}, s>3 / 2$ [18]. The system also has global strong solutions which blow up in finite time [19, 21, 22] and a global weak solution [23]. However, the problem about continuation of the solutions beyond wave breaking, although interesting and important, has not been 
explicitly addressed yet to the best of our knowledge. In our recent work [20], we studied the continuation beyond wave breaking by applying an approach based on a novel transformation that transforms system (1) into an equivalent semilinear system of original differential equation. Such treatment makes it possible to investigate the continuity of the solution beyond collision time, leading to the multipeakon conservative solution and a global conservative solution where the energy is conserved for almost all times.

It should be noted that both conservation and dissipation are important features associated with the system. The global conservation property of the $\mathrm{CH} 2$ system has been obtained in [20], and the dissipative behavior of the modified coupled two-component Camassa-Holm system, different from the $\mathrm{CH} 2$ system, has also been established in $[24,25]$; however, there is no effort made in the literature on the study of the global dissipative and the multipeakon dissipative solutions of such system studied in this paper to the best of the authors' knowledge. In this work, we explore a new approach to establish a global and stable dissipative solution of the $\mathrm{CH} 2$ system, which allows for the construction of the global dissipative multipeakon solution of this system. Our study is also motivated by the early work $[10,11]$ in the study of the global dissipative solution of the $\mathrm{CH}$ equation. The main difference is that here we deal with a coupled system and consider explicitly the mutual effect between two components, which literally makes the analysis more challenging as compared to the single one considered in $[10,11]$. The key to circumvent the difficulty is to use the skillfully defined characteristic and a new set of variables, which allows for the establishment of the global dissipative solutions of system (1). Furthermore, it is useful to understand whether or not system (1) has the multipeakon solution as with the $\mathrm{CH}$ equation [12], an important aspect related to the solutions near wave breaking. And this work develops such multipeakon solution and confirms that the semigroup of the global dissipative solution preserves the multipeakon structure.

The remainder of this paper is organized as follows. Section 2 presents the transformation from the original system to a Lagrangian semilinear system. The global solutions of the equivalent semilinear system are obtained in Section 3, which are transformed into the global dissipative solutions of the original system in Section 4. We establish the multipeakon dissipative solutions for the original system in Section 5. To demonstrate the feature of the solution, two numerical examples are considered in Section 6. Finally the paper is closed in Section 7.

\section{The Original System and the Equivalent Lagrangian System}

For simplicity, we consider here the associated evolution for positive times (of course, one would get similar results for negative times just by changing the initial condition $u_{0}$ into $\left.-u_{0}\right)$. Let us introduce an operator $\Lambda=\left(1-\partial_{x}^{2}\right)^{-1}$, which can be expressed by its associated Green's function $G=e^{-|x|} / 2$ such as $\Lambda f(x)=G * f(x)=1 / 2 \cdot \int_{R} e^{-\left|x-x^{\prime}\right|} f\left(x^{\prime}\right) d x^{\prime}$ for all $f \in L^{2}(R)$. If we define $P$ as

$$
\begin{aligned}
P(t, x) & =G *\left(u^{2}+\frac{u_{x}^{2}}{2}+\frac{v^{2}}{2}+v\right) \\
& =\frac{1}{2} \cdot \int_{R} e^{-\left|x-x^{\prime}\right|}\left(u^{2}+\frac{u_{x}^{2}}{2}+\frac{v^{2}}{2}+v\right)\left(t, x^{\prime}\right) d x^{\prime},
\end{aligned}
$$

where $v=\rho-1$, then (1) can be rewritten as

$$
\begin{gathered}
u_{t}+u u_{x}+P_{x}=0, \quad t>0, \quad x \in R, \\
v_{t}+u v_{x}+v u_{x}+u_{x}=0, \quad t>0, \quad x \in R .
\end{gathered}
$$

Moreover, for regular solutions, we have the total energy

$$
E(t)=\int_{R} u^{2}+u_{x}^{2}+v^{2} d x
$$

is constant in time. Thus (4) possesses the $H^{1}$-norm conservation law defined as

$$
\|z\|_{H^{1}}=\|u\|_{H^{1}}+\|v\|_{L^{2}}=\left(\int_{R}\left[u^{2}+u_{x}^{2}+v^{2}\right] d x\right)^{1 / 2}
$$

where $z=(u, v)$. Since $z=(u, v) \in H^{1} \times W$ with $W=L^{2} \cap L^{\infty}$, Young's inequality ensures $P \in H^{1}$.

We reformulate system (4) into a Lagrangian equivalent semilinear system as follows. Let $z(t, x)=(u, v)(t, x)$ denote the solution of system (4). For given initial data $y(0, \xi)$, we define the corresponding characteristic $y(t, \xi)$ as the solution of

$$
y_{t}(t, \xi)=u(t, y(t, \xi))
$$

where the variable $\xi$ is identified with a "particle." The change in Lagrangian energy distribution along the particle path is given by

$$
h(t, \xi)=\left(u^{2}+u_{x}^{2}+v^{2}\right) \circ y y_{\xi} .
$$

It is not hard to check that

$$
\left(u^{2}+u_{x}^{2}+v^{2}\right)_{t}+\left(u\left(u^{2}+u_{x}^{2}+v^{2}\right)\right)_{x}=\left(u^{3}-2 u P\right)_{x} .
$$

Then it follows from (7) and (9) that

$$
\frac{d h}{d t}=\left(u^{3}-2 u P\right)_{x} \circ y y_{\xi}=\left(3 U^{2}-2 P\right) U_{\xi}-2 U P_{x} y_{\xi} .
$$

Throughout the following, we use the notation

$$
\begin{gathered}
U(t, \xi)=u(t, y(t, \xi)), \quad V(t, \xi)=v(t, y(t, \xi)), \\
N(t, \xi)=u_{x}(t, y(t, \xi)) .
\end{gathered}
$$


In the following, we drop the variable $t$ for simplicity. After the change of variables $x=y(t, \xi)$ and $x^{\prime}=y\left(t, \xi^{\prime}\right)$, we obtain the following expressions for $P_{x}$ and $P$; namely,

$$
\begin{gathered}
P_{x}(\xi)=-\frac{1}{4} \cdot \int_{R} \operatorname{sgn}\left(\xi-\xi^{\prime}\right) e^{-\left|y(\xi)-y\left(\xi^{\prime}\right)\right|} \\
\times\left[h+\left(U^{2}+2 V\right) y_{\xi}\right]\left(\xi^{\prime}\right) d \xi^{\prime}, \\
P(\xi)=\frac{1}{4} \cdot \int_{R} e^{-\left|y(\xi)-y\left(\xi^{\prime}\right)\right|}\left[h+\left(U^{2}+2 V\right) y_{\xi}\right]\left(\xi^{\prime}\right) d \xi^{\prime},
\end{gathered}
$$

where we have taken that $y$ is an increasing function for any fixed time $t$ for granted (the validity will be proved later) and have used the fact that $h=\left(u^{2}+u_{x}^{2}+v^{2}\right) \circ y y_{\xi}$. From the definition of the characteristic, it follows that

$$
\begin{gathered}
U_{t}(t, \xi)=u_{t}(t, y)+u_{x}(t, y) y_{t}(t, \xi)=-P_{x} \circ y(t, \xi), \\
V_{t}(t, \xi)=v_{t}(t, y)+v_{x}(t, y) y_{t}(t, \xi) \\
=-\left[(v+1) u_{x}\right] \circ y(t, \xi), \\
N_{t}(t, \xi)=u_{x t}(t, y)+u_{x x}(t, y) y_{t}(t, \xi) \\
=\left(u^{2}-\frac{u_{x}^{2}}{2}+\frac{v^{2}}{2}+v-P\right) \circ y(t, \xi) .
\end{gathered}
$$

Let us introduce another variable $\varsigma(t, \xi)$, such as $\varsigma(t, \xi)=$ $y(t, \xi)-\xi$ (it will turn out that $\varsigma \in L^{\infty}(R)$ ). With these new variables, we now derive an equivalent system of (4):

$$
\begin{gathered}
\varsigma_{t}=U, \quad U_{t}=-P_{x}, \quad V_{t}=-(V+1) N, \\
N_{t}=U^{2}-\frac{N^{2}}{2}+\frac{V^{2}}{2}+V-P,
\end{gathered}
$$

where $P$ and $P_{x}$ are given by (12). Differentiating the first two equations in (14) with respect to $\xi$ yields

$$
\begin{gathered}
\varsigma_{\xi t}=U_{\xi}, \\
U_{\xi t}=\frac{h}{2}+\left(\frac{U^{2}}{2}+V-P\right) y_{\xi}, \\
h_{t}=\left(3 U^{2}-2 P\right) U_{\xi}-2 U P_{x} y_{\xi},
\end{gathered}
$$

which is semilinear with respect to the variables $y_{\xi}, U_{\xi}$, and $h$. Dissipative solutions differ from conservative solutions when particles collide, that is, where $y_{\xi}(t, \xi)=0$ for $\xi$ in an interval of positive length. If we solve (14) and (15), we obtain the conservative solution. However, to obtain the dissipative solution, we impose that when particles collide, they lose their energy; that is, if $y_{\xi}(\tau, \xi)=0$ for some $\tau$, then we set $h(\tau, \xi)=$ 0 . One can show that $y_{\xi}(\tau, \xi)=0$ implies $U_{\xi}(\tau, \xi)=0$ so that the system (15) implies that $y_{\xi}(t, \xi)=U_{\xi}(t, \xi)=h(t, \xi)=0$ for $t \geq \tau$. Thus, we can define $\tau(\xi)$ to be the first time when $y_{\xi}(t, \xi)$ vanishes; namely,

$$
\tau(\xi)=\sup \left\{t \in R^{+} \mid y_{\xi}\left(t^{\prime}, \xi\right)>0 \forall 0 \leq t^{\prime}<t\right\} .
$$

However, from this definition $\tau(\xi)$ in (16), it is not clear whether $\tau$ is measurable or not. We now replace this definition by the following one. Let

$$
A_{t}=\bigcup_{n \geq 1} \bigcap_{t_{i} \leq t}\left\{\xi \in R \mid y_{\xi}(t, \xi)>\frac{1}{n}\right\},
$$

where $\left\{t_{i}\right\}$ is a dense countable subset of $[0, T]$ and the sets $A_{t}$ are measurable for all $t$, with $A_{t^{\prime}} \subset A_{t}$ for $t<t^{\prime}$. Let us set $\tau^{n}(\xi)=\sum_{i=0}^{2^{n}} 2^{-n} i T \chi_{i, n}(\xi)$ with $\chi_{i, n}$ being the indicator function of the set $A_{2^{-n} i T} / A_{2^{-n}(i+1) T}$. It is not hard to know that $\tau^{n}(\xi)$ is by construction measurable and increasing with respect to $n$, which is also bounded by $T$. Thus, we define

$$
\tau(\xi)=\lim _{n \rightarrow \infty} \tau^{n}(\xi)
$$

and $\tau$ is a measurable function. One can check that this definition coincided with (16). Hence, the expressions for $P_{x}$ and $P$ in (12) become

$$
\begin{gathered}
P_{x}(\xi)=-\frac{1}{4} \cdot \int_{t<\tau(\xi)} \operatorname{sgn}\left(\xi-\xi^{\prime}\right) e^{-\left|y(\xi)-y\left(\xi^{\prime}\right)\right|} \\
\times\left[h+\left(U^{2}+2 V\right) y_{\xi}\right]\left(\xi^{\prime}\right) d \xi^{\prime} \\
P(\xi)=\frac{1}{4} \cdot \int_{t<\tau(\xi)} e^{-\left|y(\xi)-y\left(\xi^{\prime}\right)\right|}\left[h+\left(U^{2}+2 V\right) y_{\xi}\right]\left(\xi^{\prime}\right) d \xi^{\prime}
\end{gathered}
$$

and the modified system to be solved here reads

$$
\begin{gathered}
\varsigma_{t}=U, \quad U_{t}=-P_{x}, \quad V_{t}=-(V+1) N, \\
N_{t}=U^{2}-\frac{N^{2}}{2}+\frac{V^{2}}{2}+V-P, \\
\varsigma_{\xi t}=\chi_{\{t<\tau(\xi)\}} U_{\xi}, \\
U_{\xi t}=\chi_{\{t<\tau(\xi)\}}\left(\frac{h}{2}+\left(\frac{U^{2}}{2}+V-P\right) y_{\xi}\right), \\
h_{t}=\chi_{\{t<\tau(\xi)\}}\left(\left(3 U^{2}-2 P\right) U_{\xi}-2 U P_{x} y_{\xi}\right),
\end{gathered}
$$

where $\chi_{B}$ is the indicator function of the set $B$, which can be regarded as an O.D.E. in the Banach space $E=L^{\infty} \cap W \cap W \cap$ $W \cap W \cap W \cap L^{1}$.

\section{Global Solutions of the Equivalent System}

To obtain the global existence of solutions, we start from a contraction argument that offers the local existence of solutions, which is proved in the Banach space E. Note that global solutions of (20) may not exist for all initial data in $E$. However, they exist when the initial data $X_{0}=$ $\left(\varsigma_{0}, U_{0}, V_{0}, N_{0}, h_{0}\right)$ belongs to the set $\Gamma$ which is defined as follows. 
Definition 1. The set $\Gamma$ is composed of all $(\varsigma, U, V, N, h)$ such that

$$
\begin{gathered}
X=\left(\varsigma, U, V, N, \varsigma_{\xi}, U_{\xi}, h\right) \in E, \\
y_{\xi} \geq 0, \quad h \geq 0 \text { almost everywhere, } \\
y_{\xi} h=y_{\xi}^{2} U^{2}+U_{\xi}^{2}+y_{\xi}^{2} V^{2} \text { almost everywhere, } \\
\frac{1}{\left(y_{\xi}+h\right)} \in L^{\infty}(R), \\
\lim _{\xi \rightarrow-\infty} \varsigma(\xi)=0, \\
g\left(y, U, V, y_{\xi}, U_{\xi}, h\right)-1 \in W,
\end{gathered}
$$

with $y(\xi)=\varsigma(\xi)+\xi$, and $g(\mathbf{x})$ is given by

$$
g(\mathbf{x})= \begin{cases}\left|x_{5}\right|+2\left(1+x_{2}^{2}+x_{3}^{2}\right) x_{4}, & \text { if } x \in \Omega, \\ x_{4}+x_{6}, & \text { otherwise }\end{cases}
$$

for $\mathbf{x}=\left(x_{1}, x_{2}, x_{3}, x_{4}, x_{5}, x_{6}\right) \in R^{6}$, where $\Omega$ is the following subset of $R^{6}$ :

$$
\begin{aligned}
\Omega= & \left\{\mathbf{x} \in R^{6}|| x_{5} \mid+2\left(1+x_{2}^{2}+x_{3}^{2}\right) x_{4} \leq x_{4}+x_{6}\right. \\
& \text { and } \left.x_{5} \leq 0\right\} .
\end{aligned}
$$

We write system (21) in a compact form that

$$
Y_{t}(t, \xi)=\chi_{\{t<\tau(\xi)\}}(\xi) F(X) Y(t, \xi)
$$

where $Y=\left(\varsigma_{\xi}, U_{\xi}, h\right)$. For $t>\tau$, we set $Y(t, \xi)=Y(\tau(\xi), \xi)$. Note that, in this definition, we do not reset the energy density $h$ to zero after collision but keep the value it reached just before the collision. Let us define $B_{M}$ (the subset of $E$ ) as follows:

$$
\begin{aligned}
B_{M}=\left\{X=\left(\varsigma, U, V, N, \varsigma_{\xi}, U_{\xi}, h\right) \in E \mid\|X\|_{E}\right. \\
\left.+\|g(X)-1\|_{W}+\left\|\frac{1}{\left(y_{\xi}+h\right)}\right\|_{L_{T}^{\infty} L_{R}^{\infty}} \leq M\right\} .
\end{aligned}
$$

Theorem 2. Given initial data $X_{0}=\left(\varsigma_{0}, U_{0}, V_{0}, N_{0}, h_{0}\right) \in \Gamma$, there exists a time $T>0$ such that the system (20) and (21) admit a unique solution in $C^{1}\left([0, T], B_{M}\right)$.

Proof. To obtain the local existence of solutions, it suffices to show that $F(X)$ given by (25) is a contraction, and, therefore, there exists a unique fixed point $\left(\varsigma, U, V, N, \varsigma_{\xi}, U_{\xi}, h\right) \in$ $C\left([0, T], B_{M}\right)$ which is solution to (20) and (21).
Our main task is to prove the estimates for $P$ and $P_{x}$ defined by (19). Let us write $P_{x}(\xi)$ as $P_{x}(\xi)=P_{x}^{1}(\xi)+P_{x}^{2}(\xi)$ with

$$
\begin{gathered}
P_{x}^{1}(\xi)=-\frac{e^{-\zeta(\xi)}}{4} \cdot\left(f_{1} *\left[\chi_{\{t<\tau(\xi)\}} r_{1}\right]\right)(t, \xi), \\
P_{x}^{2}(\xi)=\frac{e^{\zeta(\xi)}}{4} \cdot\left(f_{2} *\left[\chi_{\{t<\tau(\xi)\}} r_{2}\right]\right)(t, \xi),
\end{gathered}
$$

where

$$
\begin{gathered}
f_{1}(\xi)=\chi_{\{\xi>0\}}(\xi) e^{-\xi}, \quad r_{1}=r_{1}^{1}+r_{1}^{2}, \\
r_{1}^{1}=e^{\varsigma}\left(U^{2}+2 V\right)\left(1+\varsigma_{\xi}\right), \quad r_{1}^{2}=e^{\varsigma} h, \\
f_{2}(\xi)=\chi_{\{\xi<0\}}(\xi) e^{-\xi}, \quad r_{2}=r_{2}^{1}+r_{2}^{2}, \\
r_{2}^{1}=e^{-\varsigma}\left(U^{2}+2 V\right)\left(1+\varsigma_{\xi}\right), \quad r_{2}^{2}=e^{-\varsigma} h .
\end{gathered}
$$

Young's inequalities imply that

$$
\begin{aligned}
& \left\|P_{x}^{1}(t, \cdot)\right\|_{W} \\
& \leq \frac{1}{4} \cdot\left\|e^{-\varsigma}\right\|_{L_{R}^{\infty}} \\
& \quad \times\left(\left\|f_{1} *\left[\chi_{\{t<\tau(\xi)\}} r_{1}^{1}\right]\right\|_{W}+\left\|f_{1} *\left[\chi_{\{t<\tau(\xi)\}} r_{1}^{2}\right]\right\|_{W}\right) \\
& \leq C(M)\left(\left(\left\|f_{1}\right\|_{L_{R}^{1}}+\left\|f_{1}\right\|_{L_{R}^{2}}\right)\left\|r_{1}^{1}\right\|_{L_{R}^{2}}\right. \\
& \left.\quad+\left(\left\|f_{1}\right\|_{L_{R}^{2}}+\left\|f_{1}\right\|_{L_{R}^{\infty}}\right)\left\|r_{1}^{2}\right\|_{L_{R}^{1}}\right) \leq C(M),
\end{aligned}
$$

where we have denoted by $C(M)$ a generic constant. The estimate for $P$ can be obtained in exactly the same way. We thus obtain that $F(X)$ given in (25) is a contraction, and the local existence of solutions follows from the standard contraction argument of ordinary differential equations.

When proving existence and stability, a priori control is essential. Let us introduce the set

$$
\begin{aligned}
A_{\varepsilon}= & \left\{\xi \in R \mid 0<y_{0, \xi}(\xi) \leq \varepsilon,-\varepsilon \leq U_{0, \xi}(\xi)<0\right. \\
& \text { or } \left.-\varepsilon \leq V_{0, \xi}(\xi)<0\right\},
\end{aligned}
$$

and therefore there exists an $\varepsilon(\bar{M}, T)$ such that

$$
0<y_{\xi}(\xi) \leq \varepsilon, \quad-\varepsilon \leq U_{\xi}(\xi)<0,
$$

for $\xi \in A_{\varepsilon}$, and in addition

$$
A_{\varepsilon} \subset\{\xi \in R \mid 0<\tau(\xi)<T\},
$$

and the set

$$
K_{\gamma}=\left\{\xi \in R \mid h_{0}(\xi) \geq \gamma\right\}
$$


and therefore, by taking $\gamma$ large enough depending only on $\bar{M}$ and $T, y_{\xi}(t, \xi)$ is a decreasing function and $U_{\xi}(t, \xi)$ are increasing functions of time for $\xi \in K_{\gamma}$, and in addition

$$
K_{\gamma} \subset\{\xi \in R \mid 0<\tau(\xi)<T\} .
$$

We have $\lim _{\varepsilon \rightarrow 0}$ meas $\left(A_{\varepsilon}\right)=0$ because $\bigcap_{n>0} A_{1 / n}=\emptyset$, and $\operatorname{meas}\left(K_{\gamma}\right) \leq 1 / \gamma \cdot \int_{R} h_{0}(\xi) d \xi \leq 1 / \gamma \cdot\left\|h_{0}\right\|_{L_{R}^{1}}$ which implies $\lim _{\varepsilon \rightarrow 0} \operatorname{meas}\left(K_{\gamma}\right)=0$.

Lemma 3. Given $X_{0} \in B_{M_{0}}$ for some constant $M_{0}$, one denotes the solution of (20), (21) by $X=\left(\varsigma, U, V, N, \varsigma_{\xi}, U_{\xi}, V_{\xi}, N_{\xi}, h\right) \in$ $C\left([0, T], B_{M}\right)$ with initial data $X_{0}$. Let $\bar{M}=\|P\|_{L_{T}^{\infty} L_{R}^{\infty}}+$ $\left\|P_{x}\right\|_{L_{T}^{\infty} L_{R}^{\infty}}+M_{0}$. Then the following hold.

(i) Consider

$$
\begin{gathered}
y_{\xi}(t, \xi) \geq 0, \quad h(t, \xi) \geq 0, \\
y_{\xi} h=y_{\xi}^{2} U^{2}+U_{\xi}^{2}+y_{\xi}^{2} V^{2}
\end{gathered}
$$

for all $t$ and almost all $\xi$. Thus $y_{\xi}(t, \xi)=0$ implies $U_{\xi}(t, \xi)=0$.

(ii) Consider

$$
\left\|\frac{1}{\left(y_{\xi}+h\right)}(t, \cdot)\right\|_{L_{R}^{\infty}} \leq \frac{9}{2} \cdot e^{C(\bar{M}, T) T}\left\|\frac{1}{\left(y_{\xi}+h\right)}(0, \cdot)\right\|_{L_{R}^{\infty}}
$$

for all $t \in[0, T]$ and a constant $C(M, T)$.

Proof. (i) We know that (35)-(37) hold for almost every $\xi \in R$ at $t=0$ because $X_{0} \in \Gamma$. We consider a fixed $\xi$ that we suppress in the notation. On the one hand, it follows from (21) that

$$
\begin{aligned}
\left(y_{\xi} h\right)_{t} & =U_{\xi} h+y_{\xi}\left[\left(3 U^{2}-2 P\right) U_{\xi}-2 U P_{x} y_{\xi}\right] \\
& =U_{\xi} h+3 U^{2} U_{\xi} y_{\xi}-2 P U_{\xi} y_{\xi}-2 U P_{x} y_{\xi}^{2},
\end{aligned}
$$

and on the other hand

$$
\begin{aligned}
\left(y_{\xi}^{2} U^{2}+\right. & \left.U_{\xi}^{2}+y_{\xi}^{2} V^{2}\right)_{t} \\
= & 2 y_{\xi} y_{\xi t} U^{2}+2 y_{\xi}^{2} U U_{t} \\
& +2 y_{\xi} y_{\xi t} V^{2}+2 y_{\xi}^{2} V V_{t}+2 U_{\xi} U_{\xi t} \\
= & 3 U^{2} U_{\xi} y_{\xi}-2 U P_{x} y_{\xi}^{2}+U_{\xi} h-2 P U_{\xi} y_{\xi} .
\end{aligned}
$$

Hence, $\left(y_{\xi} h\right)_{t}=\left(y_{\xi}^{2} U^{2}+U_{\xi}^{2}+y_{\xi}^{2} V^{2}\right)_{t}$, which together with $y_{\xi} h(0)=\left(y_{\xi}^{2} U^{2}+U_{\xi}^{2}+y_{\xi}^{2} V^{2}\right)(0)$ yields $y_{\xi} h(t)=\left(y_{\xi}^{2} U^{2}+\right.$ $\left.U_{\xi}^{2}+y_{\xi}^{2} V^{2}\right)(t)$ for all $t \in[0, T]$, and (36) has been proved. It follows directly from the definition of $\tau$ and $y_{\xi}$ that $y_{\xi}(t) \geq 0$ for $t \geq 0$. For $t \in[0, \tau)$, we have $h(t) \geq 0$ as $h(t)=\left(y_{\xi}^{2} U^{2}+U_{\xi}^{2}+\right.$ $\left.y_{\xi}^{2} V^{2}\right) / y_{\xi}(t)$. The continuity (with respect to $t$ ) of $h$ implies $h(\tau(\xi)) \geq 0$ and, therefore, $h(t) \geq 0$ for all $t \geq 0$ because the variable does not change for $t \geq \tau(\xi)$. (ii) We consider a fixed $\xi$ and drop it in the notation. Let us denote the Euclidean norm of $Y=\left(y_{\xi}, U_{\xi}, h\right)$ by $|Y|_{2}=$ $\left(y_{\xi}^{2}+U_{\xi}^{2}+h^{2}\right)^{1 / 2}$. Since $Y_{t}=F(X) Y$, we have

$$
\begin{aligned}
\frac{d}{d t} \cdot|Y|_{2}^{-2} & =-2|Y|_{2}^{-4} Y \cdot \frac{d Y}{d t} \\
& =-2|Y|_{2}^{-4} Y \cdot F(X) Y \\
& \leq 2 \sup _{t \in[0, T]}|F(X)(t)||Y|_{2}^{-2} .
\end{aligned}
$$

We have $|F(X)(t)| \leq K\left(\|P\|_{L_{T}^{\infty} L_{R}^{\infty}}+\left\|P_{x}\right\|_{L_{T}^{\infty} L_{R}^{\infty}}+\|U\|_{L_{T}^{\infty} L_{R}^{\infty}}+\right.$ $\left.\|V\|_{L_{T}^{\infty} L_{R}^{\infty}}\right)$ for a constant $K$ depending on the norm chosen for the matrix $F$. We infer from (25) that

$$
\|U\|_{L_{T}^{\infty} L_{R}^{\infty}} \leq U_{0}+T\left\|P_{x}\right\|_{L_{T}^{\infty} L_{R}^{\infty}} \leq C(\bar{M}, T),
$$

for a constant depending only on $\bar{M}$ and $T$. We denote generically by $C(\bar{M}, T)$ such constant. Thus, $|F(X)(t)| \leq C(M, T)$. Gronwall's lemma implies that $|Y(t)|_{2}^{-2} \leq e^{2 C(\bar{M}, T) T}|Y(0)|_{2}^{-2}$, and therefore

$$
\frac{1}{\left(\left|y_{\xi}\right|+\left|U_{\xi}\right|+|h|\right)}(t) \leq \frac{3}{\left(\left|y_{\xi}\right|+\left|U_{\xi}\right|+|h|\right)}(0) \cdot e^{C(\bar{M}, T) T} .
$$

Since $y_{\xi}>0$ and $h>0$, it follows from (36) that $\left|U_{\xi}(t)\right| \leq$ $\sqrt{y_{\xi}(t) h(t)} \leq\left(y_{\xi}+h\right)(t) / 2$, and therefore $\left(\left|y_{\xi}\right|+\left|U_{\xi}\right|+|h|\right)(t) \leq$ $3 / 2 \cdot\left(y_{\xi}+h\right)(t)$, which yields

$$
\frac{1}{\left(\left|y_{\xi}\right|+|h|\right)}(t) \leq \frac{9}{2} \cdot e^{C(\bar{M}, T) T} \cdot \frac{1}{\left(\left|y_{\xi}\right|+|h|\right)}(0) .
$$

We show that the local solution can be extended into the global solution.

Theorem 4. Given initial data $\left(y_{0}, U_{0}, V_{0}, N_{0}, h_{0}\right) \in \Gamma$, system (20) and (21) admit a unique global solution $(y, U, V, N, h) \in$ $C\left(R^{+}, \Gamma\right)$.

Proof. The local solution described in Theorem 2 does not provide any lower bound on the time of existence of the solution. Let us introduce the maximum time of existence $T_{\max }$ defined as

$$
\begin{gathered}
T_{\max }=\sup \left\{t \in R^{+} \mid \text {the solutions } X(t) \text { to }(20)\right. \\
\text { exists in }[0, t]\} .
\end{gathered}
$$

Let us assume that $T_{\max }<\infty$. To prove global existence of the solution, the basic ingredient is a global bound on the solution $X$.

We begin by showing that, for given $M_{0}, T_{0}>0$, there exists a constant $M\left(M_{0}, T_{0}\right)$ such that, for any $X_{0} \in B_{M_{0}}$, if $h_{0} \in W(B)$ for some set $B$, then $\|X\|_{L_{T}^{\infty} W(B)}$ is bounded by a constant $C\left(M,\left\|h_{0}\right\|_{W(B)}\right)$. It follows from (21) that

$$
\begin{aligned}
& \|h(t, \cdot)\|_{L^{1}} \\
& \quad=\lim _{N \rightarrow \infty} \int_{-N}^{N} h(t, \xi) d \xi
\end{aligned}
$$




$$
\begin{aligned}
& =\lim _{N \rightarrow \infty}\left(\int_{-N}^{N} h(0, \xi) d \xi\right. \\
& \quad+\int_{0}^{t}\left(\left(U^{3}-2 P U\right)\left(t^{\prime}, N\right)\right. \\
& \left.\left.-\left(U^{3}-2 P U\right)\left(t^{\prime},-N\right)\right) d t^{\prime}\right) \\
& =\lim _{N \rightarrow \infty} \int_{-N}^{N} h(0, \xi) d \xi=\|h(0, \cdot)\|_{L^{1}},
\end{aligned}
$$

for all $t \in[0, T]$, where we have used that $\lim _{\xi \rightarrow \pm \infty} U(t, \xi)=$ 0 as $U(t, \cdot) \in H^{1}(R)$. We denote by $M\left(M_{0}, T_{0}\right)$ a general constant and drop $t$ for simplification. We have

$$
\begin{aligned}
U^{2}(\xi) & =2 \int_{-\infty}^{\xi} U(\eta) U_{\xi}(\eta) d \eta \leq \int_{R} U(\eta) U_{\xi}(\eta) d \eta \\
& =\int_{\left\{y_{\xi}(\eta)>0\right\}} U(\eta) U_{\xi}(\eta) d \eta \\
& \leq \frac{1}{2} \cdot \int_{\left\{y_{\xi}(\eta)>0\right\}}\left(y_{\xi} U^{2}+\frac{U_{\xi}^{2}}{y_{\xi}}\right)(\eta) d \eta \\
& =\frac{1}{2} \cdot \int_{\left\{y_{\xi}(\eta)>0\right\}} h(\eta) d \eta \leq \frac{1}{2}\|h(t, \cdot)\|_{L^{1}(R)},
\end{aligned}
$$

where we have used the fact that when $y_{\xi}(\xi)=0, U_{\xi}(\xi)=0$. Thus,

$$
\begin{aligned}
\|U(t, \cdot)\|_{L^{\infty}(R)} & \leq \frac{1}{\sqrt{2}}\|h(t, \cdot)\|_{L^{1}(R)}^{1 / 2} \\
& =\frac{1}{\sqrt{2}}\left\|h_{0}\right\|_{L^{1}(R)}^{1 / 2} \leq M\left(M_{0}, T_{0}\right) .
\end{aligned}
$$

We can obtain from the governing equation (20) that

$$
|\varsigma(t, \xi)| \leq|\varsigma(0, \xi)|+\|U(t, \cdot)\|_{L^{\infty},} \quad T \leq M\left(M_{0}, T_{0}\right),
$$

and then $\|\zeta(t, \cdot)\|_{L_{R}^{\infty}} \leq M\left(M_{0}, T_{0}\right)$. We can also get from the governing equation (20) that

$$
\begin{gathered}
\|V(t, \cdot)\|_{L^{\infty}(R)} \leq M\left(M_{0}, T_{0}\right), \\
\|N(t, \cdot)\|_{L_{R}^{\infty}} \leq M\left(M_{0}, T_{0}\right) .
\end{gathered}
$$
that

From the identity $h=\left(U^{2}+M^{2}+V^{2}\right) y_{\xi}$, we can deduce

$$
\left|\left(U^{2}+2 V\right) y_{\xi}\right| \leq\left(U^{2}+V^{2}+1\right) y_{\xi} \leq h+y_{\xi},
$$

which implies that

$$
\begin{aligned}
\left|P_{x}\right| & \leq \frac{1}{4} \cdot\left|\int_{R} e^{-\left|y(\xi)-y\left(\xi^{\prime}\right)\right|}\left[h+\left(U^{2}+2 V\right) y_{\xi}\right]\left(\xi^{\prime}\right) d \xi^{\prime}\right| \\
& \leq \frac{1}{4} \cdot\left|\int_{R} e^{-\left|y(\xi)-y\left(\xi^{\prime}\right)\right|}\left[2 h+y_{\xi}\right]\left(\xi^{\prime}\right) d \xi^{\prime}\right| \\
& \leq C\left(\|h(t, \cdot)\|_{L^{1}}+\|\varsigma(t, \cdot)\|_{L_{R}^{\infty}}\right) \leq M\left(M_{0}, T_{0}\right) .
\end{aligned}
$$

Therefore, $\left\|P_{x}\right\|_{L^{\infty}} \leq M\left(M_{0}, T_{0}\right)$. To summarize, we have established

$$
\begin{aligned}
\|\varsigma(t, \cdot)\|_{L_{R}^{\infty}}+\|U(t, \cdot)\|_{L_{R}^{\infty}}+\|V(t, \cdot)\|_{L_{R}^{\infty}} \\
\quad+\|N(t, \cdot)\|_{L_{R}^{\infty}}+\|h(t, \cdot)\|_{L_{R}^{\infty}}+\|P(t, \cdot)\|_{L_{R}^{\infty}} \\
\quad+\left\|P_{x}(t, \cdot)\right\|_{L_{R}^{\infty}} \leq M\left(M_{0}, T_{0}\right) .
\end{aligned}
$$

We consider a set $B$ such that $\left\|h_{0}\right\|_{W(B)}$ is finite. Thus it is not hard to obtain that

$$
\begin{aligned}
\left\|P_{x}\right\|_{L^{2}(B)} & \leq\left(\|h(t, \cdot)\|_{L^{1}(B)}+\|\varsigma(t, \cdot)\|_{L^{\infty}(B)}\right)\left\|e^{-y(\xi)}\right\|_{L^{2}(B)} \\
& \leq M\left(M_{0}, T_{0}\right) .
\end{aligned}
$$

Similarly, one can obtain that the same bound holds for $P$. Let

$$
\begin{aligned}
\alpha(t)= & \|U(t, \cdot)\|_{W(B)}+\left\|U_{\xi}(t, \cdot)\right\|_{W(B)} \\
& +\|V(t, \cdot)\|_{W(B)}+\left\|\varsigma_{\xi}(t, \cdot)\right\|_{W(B)}+\|h(t, \cdot)\|_{W(B)} .
\end{aligned}
$$

By taking the $W(B)$-norm on both sides of (20) and (21), it follows that

$$
\alpha(t) \leq \alpha(0)+M\left(M_{0}, T_{0}\right) \int_{0}^{t} \alpha\left(t^{\prime}\right) d t^{\prime},
$$

which, by using Gronwall's lemma, yields

$$
\alpha(t) \leq \alpha(0) e^{M\left(M_{0}, T_{0}\right) T} \leq C
$$

for some constant $C$ depending only on $M\left(M_{0}, T_{0}\right)$ and $\left\|h_{0}\right\|_{W(B)}$.

It is not hard to check that there exists a constant $M$ such that $X(t) \in B_{M}$ for all $t \in\left[0, T_{\max }\right)$. We know that there exists a constant $\gamma$ such that (34) holds. From (20), we get

$$
\begin{gathered}
\left\|\varsigma\left(t^{\prime}, \cdot\right)-\varsigma(t, \cdot)\right\|_{L^{\infty}(R)} \leq M\left|t^{\prime}-t\right|, \\
\left\|U\left(t^{\prime}, \cdot\right)-U(t, \cdot)\right\|_{W} \leq C(M)\left|t^{\prime}-t\right|, \\
\left\|V\left(t^{\prime}, \cdot\right)-V(t, \cdot)\right\|_{W} \leq C(M)\left|t^{\prime}-t\right|, \\
\left\|N\left(t^{\prime}, \cdot\right)-N(t, \cdot)\right\|_{W} \leq C(M)\left|t^{\prime}-t\right|,
\end{gathered}
$$

for a constant $C(M)$ depending only on $M$. We denote generically by $C(M)$ such constants. From the above proof, we have $\|h(t, \cdot)\|_{W\left(K_{\gamma}^{c}\right)} \leq C(M)$. It follows from (25) that

$$
\begin{aligned}
& \left\|Y\left(t^{\prime}, \cdot\right)-Y(t, \cdot)\right\|_{W\left(K_{\gamma}^{c}\right)} \\
& \quad \leq \int_{t}^{t^{\prime}}\|F(X) Y\|_{W\left(K_{\gamma}^{c}\right)} \leq C(M)\left|t^{\prime}-t\right| .
\end{aligned}
$$


We obtain from $(22 c)$ that

$$
\begin{aligned}
& \left\|h\left(t^{\prime}, \cdot\right)-h(t, \cdot)\right\|_{L^{1}\left(K_{\gamma}^{c}\right)} \\
& =\|\left[U^{2}\left(1+\varsigma_{\xi}\right)^{2}+U_{\xi}^{2}+V^{2}\left(1+\varsigma_{\xi}\right)^{2}-\varsigma_{\xi} h\right]\left(t^{\prime}, \cdot\right) \\
& -\left[U^{2}\left(1+\varsigma_{\xi}\right)^{2}+U_{\xi}^{2}+V^{2}\left(1+\varsigma_{\xi}\right)^{2}-\varsigma_{\xi} h\right](t, \cdot) \|_{L^{1}\left(K_{\gamma}^{c}\right)} \\
& \leq C(M)\left(\left\|U\left(t^{\prime}, \cdot\right)-U(t, \cdot)\right\|_{L^{2}\left(K_{\gamma}^{c}\right)}\right. \\
& +\left\|V\left(t^{\prime}, \cdot\right)-V(t, \cdot)\right\|_{L^{2}\left(K_{\gamma}^{c}\right)} \\
& \left.+\left\|Y\left(t^{\prime}, \cdot\right)-Y(t, \cdot)\right\|_{L^{2}\left(K_{\gamma}^{c}\right)}\right) \\
& \leq C(M)\left|t^{\prime}-t\right|
\end{aligned}
$$

and, therefore, $\left\|X\left(t^{\prime}, \cdot\right)-X(t, \cdot)\right\|_{E\left(K_{\gamma}^{c}\right)} \leq C(M)\left|t^{\prime}-t\right|$. For $\xi \in$ $K_{\gamma}$, we have from $(34)$ that $\left(\varsigma_{\xi}, U_{\xi}, h\right)\left(t^{\prime}, \xi\right)=\left(\varsigma_{\xi}, U_{\xi}, h\right)(t, \xi)$ for $t^{\prime} \geq t \geq T$. Thus we have

$$
\left\|X\left(t^{\prime}, \cdot\right)-X(t, \cdot)\right\|_{E} \leq C(M)\left|t^{\prime}-t\right|,
$$

which implies that $\lim _{t \rightarrow T_{\max }} X(t)$ exists as $E$ is a Banach space and we denote it by $\widetilde{X}$. We claim that $\widetilde{X} \in \Gamma$. Since $X(t) \in$ $B_{M}$ for all $t \in\left[0, T_{\max }\right)$, we have $\left\|1 /\left(y_{\xi}+h\right)(t, \cdot)\right\| \leq M$ and therefore $\left\|1 /\left(\tilde{y}_{\xi}+\widetilde{h}\right)\right\| \leq M$ and the condition (22d) holds. Since $L^{\infty}$ or $L^{1}$ convergence implies almost everywhere convergence up to a subsequence, the conditions (22b), (22c), and (22e) are fulfilled. It remains to check (22f). Note that the mapping $g$ defined in (23) is lower-semicontinuous. We consider a sequence $t_{n} \rightarrow T_{\max }$ such that $X\left(t_{n}, \xi\right) \rightarrow \widetilde{X}(\xi)$ for almost every $\xi$. We can check from the definition of $g$ that $g(X)-y_{\xi}$ is positive as $y_{\xi}$ and $h$ are positive. Thus, we get by the lower semicontinuity of $g$ that

$$
\begin{aligned}
0 & \leq g(\widetilde{X}(\xi))-\tilde{y}_{\xi}(\xi) \\
& \leq \lim \inf \left(g\left(X\left(t_{n}, \cdot\right)\right)-y_{\xi}\left(t_{n}, \cdot\right)\right) \leq 2 M
\end{aligned}
$$

and $g(\widetilde{X})-1 \in L^{\infty}(R)$ because $\|g(\widetilde{X})-1\|_{L^{\infty}(R)} \leq$ $\left\|g(\widetilde{X})-\widetilde{y}_{\xi}\right\|_{L^{\infty}(R)}+\left\|\widetilde{\zeta}_{\xi}\right\|_{L^{\infty}(R)} \leq 3 M$. The composition of an increasing lower semicontinuous function with a lower semicontinuous function is also lower semicontinuous. Hence, since $z \rightarrow z^{2}$ is increasing for $z \geq 0$ and $g(X)-y_{\xi}$ is positive, we get from Fatou's Lemma that

$$
\begin{aligned}
\int_{R}\left|g(\widetilde{X}(\xi))-\tilde{y}_{\xi}(\xi)\right|^{2} d \xi \\
\quad \leq \int_{R} \lim \inf \left|g\left(X\left(t_{n}, \xi\right)\right)-y_{\xi}\left(t_{n}, \xi\right)\right|^{2} d \xi \\
\quad \leq \lim \inf \int_{R}\left|g\left(X\left(t_{n}, \xi\right)\right)-y_{\xi}\left(t_{n}, \xi\right)\right|^{2} d \xi
\end{aligned}
$$

which implies

$$
\|g(\widetilde{X})-1\|_{L^{2}(R)} \leq\left\|g(\widetilde{X})-\widetilde{y}_{\xi}\right\|_{L^{2}(R)}+\left\|\widetilde{\varsigma}_{\xi}\right\|_{L^{2}(R)} \leq 3 M
$$

as

$$
\begin{aligned}
& \left\|g\left(X\left(t_{n}, \cdot\right)\right)-y_{\xi}\left(t_{n}, \cdot\right)\right\|_{L^{2}(R)} \\
& \quad \leq\left\|g\left(X\left(t_{n}, \cdot\right)\right)-1\right\|_{L^{2}(R)}+\left\|\zeta_{\xi}\left(t_{n}, \cdot\right)\right\|_{L^{2}(R)} \leq 2 M .
\end{aligned}
$$

Hence, $\widetilde{X}$ fulfills (22f) and $\widetilde{X} \in \Gamma$. We get from Theorem 2 the existence of a local solution with initial data $\widetilde{X}$ which, combined with $X$ on $\left[0, T_{\max }\right)$, gives a solution on $\left[0, T_{\max }+\right.$ $\delta$ ) for some $\delta>0$. The assumption regarding $T_{\max }$ is contradicted, and we have proved the global existence of solutions.

\section{Global Dissipative Solutions for the Original System}

We show that the global solution of the equivalent system (20) and (21) yields a global dissipative solution of the original system (4), which needs to establish the correspondence between the Lagrangian equivalent system and the original system.

Let us start by introducing the set $\Gamma_{0}$ as $\Gamma_{0}=\{X \in \Gamma \mid$ $g(X)=1\}$. For any $X \in \Gamma$, we define

$$
\varphi(\xi)=\int_{-\infty}^{\xi}\left(g(X(\eta))-y_{\xi}(\eta)\right) d \eta+y(\xi)
$$

so that $\varphi_{\xi}=g(X)$, and

$$
\begin{array}{ll}
\bar{y}(\xi)=y(\eta), & \bar{U}(\xi)=U(\eta), \\
\bar{V}(\xi)=V(\eta), & \bar{N}(\xi)=N(\eta)
\end{array}
$$

for any $\eta$ such that $\xi=\varphi(\eta)$. We consider the pushforward of $h d \xi$ by $\varphi$ and denote it by $v$; that is, $v=\varphi_{\#}(h d \xi)$. By the Radon-Nikodym theorem, there exists a unique function $\bar{h}$ in $L^{1}$ such that

$$
\varphi_{\#}(h d \xi)=v=\bar{h} d \xi+v_{s},
$$

where $\nu_{s}$ is the singular part of the decomposition of $\nu$ and $\bar{h} d \xi$ the absolutely continuous part. From the definitions (65)(67), it is not hard to check that $\bar{X}=(\bar{\varsigma}, \bar{U}, \bar{V}, \bar{N}, \bar{h}) \in \Gamma_{0}$. Thus we define the mapping $\Pi: \Gamma \rightarrow \Gamma_{0}$ as $\bar{X}=\prod(X)$, which is a continuous mapping with respect to the distance $\widetilde{d}_{R}$ on bounded set of $\Gamma$; that is, for any sequence $X^{n}$ and $X$ in $B_{M}$, we have

$$
\lim _{n \rightarrow \infty} \tilde{d}_{R}\left(X^{n}, X\right)=0 \quad \text { implies } \lim _{n \rightarrow \infty} \tilde{d}_{R}\left(\prod\left(X^{n}\right), \prod(X)\right)=0 \text {. }
$$

The system is invariant with respect to relabeling. Let us explain what we mean by relabeling. 
Definition 5. If there exists a $\psi$ which satisfies

$$
\begin{gathered}
\psi(\xi)-\xi \in L^{\infty}(R), \\
\psi_{\xi}-1 \in L^{\infty}(R) \cap L^{2}(R), \quad \psi_{\xi} \geq 0, \\
\lim _{\xi \rightarrow-\infty}(\psi(\xi)-\xi)=0
\end{gathered}
$$

and such that

$$
\bar{y}=y \circ \psi, \quad \bar{U}=U \circ \psi, \quad \bar{V}=V \circ \psi, \quad \bar{N}=N \circ \psi,
$$

one says that $\bar{X} \in \Gamma$ is a relabeling of $X \in \Gamma$.

Note that $\bar{X} \in \Gamma$ and $X \in \Gamma$ imply that for almost all $\xi \in R$ such that $\bar{y}_{\xi}(\xi) \neq 0$,

$$
\bar{h}(\xi)=h \circ \psi \psi_{\xi}(\xi)
$$

Thus, for any $t \geq 0$, if $\bar{X}_{0}$ is a relabeling of $X_{0}, \bar{X}(t)=S_{t}\left(\bar{X}_{0}\right)$ is a relabeling of $X(t)=S_{t}\left(X_{0}\right)$. In the dissipative case, we cannot define an equivalence relation between elements that are equal up to a relabeling. If $\bar{X}$ is a relabeling of $X$, then $X$ is not necessarily a relabeling of $\bar{X}$, basically because $\psi^{-1}$ is either not well defined or not sufficiently regular. However, we have the result that if $X_{2}$ is a relabeling of $X_{1}$, then $\Pi\left(X_{2}\right)=$ $\Pi\left(X_{1}\right)$. Thus we can define an equivalence relation in $\Gamma$ as follows: $X_{1}$ and $X_{2}$ are equivalent if $\prod\left(X_{1}\right)=\prod\left(X_{2}\right)$; that is, if $X_{2}$ is a relabeling of $X_{1}$, then $X_{1}$ and $X_{2}$ are equivalent. The set of equivalent classes is in bijection with $\Gamma_{0}$ on which we can define a semigroup as $\widetilde{S}_{t}=\prod \circ S_{t}$ with the semigroup property

$$
\widetilde{S}_{t+t^{\prime}}=\prod \circ S_{t+t^{\prime}}=\prod \circ S_{t} \circ S_{t^{\prime}}=\prod \circ S_{t} \circ \prod \circ S_{t^{\prime}}=\widetilde{S}_{t} \circ \widetilde{S}_{t^{\prime}} .
$$

We now establish a bijection between Lagrangian equivalent system and original system by introducing two mappings between the original variable $z=(u, v) \in H^{1} \times W$ and the Lagrangian variable $X \in \Gamma_{0}$.

The mapping $L$ is defined from original system to Lagrangian equivalent system as follows. Given $(u, v) \in H^{1} \times$ $W$, we denote $\bar{X}(x)=\left(x, u, v, 1, u_{x}, u^{2}+u_{x}^{2}+v^{2}\right)$ and define $y$ as

$$
\int_{-\infty}^{y(\xi)}(g(\bar{X}(x))-1) d x+y(\xi)=\xi
$$

and set

$$
(U, V, N)=\left(u, v, u_{x}\right) \circ y, \quad h=\left(u^{2}+u_{x}^{2}+v^{2}\right) \circ y y_{\xi} .
$$

Then, $X=(y, U, V, N, h) \in \Gamma_{0}$. We denote by $L$ the mapping $(u, v) \rightarrow X$ from $H^{1} \times W$ to $\Gamma_{0}$, which sends bounded set of $H^{1} \times W$ into bounded sets of $\Gamma_{0}$; that is, for any $M>0$ and $(u, v) \in H^{1} \times W,\|z\|_{H^{1}} \leq M$ implies $L(z) \in B_{\bar{M}}$ for some constant $\bar{M}$ depending only on $M$.
Let us introduce the mapping $M$ from Lagrangian equivalent system to original system. Given $X \in \Gamma$, if $x=y(\xi)$, the function

$$
(u, v)(x)=(U, V)(\xi)
$$

is well defined and belongs to $H^{1} \times W$. We denote by $M$ the mapping $X \rightarrow(u, v)$ from $\Gamma_{0}$ to $H^{1} \times W$ with the property that $M \circ \prod=M$ from which we know that $\prod(X)=\prod(\bar{X})$ implies $M(X)=M(\bar{X})$.

We claim that $H^{1} \times W$ is in bijection with $\Gamma_{0}$ as the following theorem shows.

Theorem 6. Consider the following

$$
M \circ L=I d, \quad L \circ M=\prod \text {. }
$$

Proof. Given $(u, v) \in H^{1} \times W$, we denote $X=L(u, v)$ and $(\bar{u}, \bar{v})=M(X)$. We have $(U, V)=(u, v) \circ y$. Since $X \in \Gamma_{0}, y$ is invertible and therefore $(\bar{u}, \bar{v})=(U, V) \circ y^{-1}$. Hence, $(\bar{u}, \bar{v})=$ $(u, v)$ and $M \circ L=I d$.

Given $X \in \Gamma_{0}$, we denote $(u, v)=M(X)$ and $\bar{X}=L(u, v)$. Let $S=\{\xi \in R \mid X(\xi) \in \Omega\}$. It is not hard to check that meas $(S)<\infty$. As we have known, $g(X)-y_{\xi} \in L^{1}$ and since $g(X)=1$, we have $g(X)-y_{\xi}=1-y_{\xi}=-\varsigma_{\xi}$ and $\int_{\xi^{\prime}}^{\xi}(g(X)-$ $\left.y_{\xi}\right) d \eta=-\varsigma(\xi)+\varsigma\left(\xi^{\prime}\right)$. Let $\xi^{\prime} \rightarrow-\infty$. We get

$$
\int_{-\infty}^{\xi}\left(g(X)-y_{\xi}\right) d \eta+y(\xi)=\xi
$$

as $\lim _{\xi \rightarrow-\infty} \varsigma(\xi)=0$, which can be rewritten as

$$
\begin{aligned}
\int_{-\infty}^{\xi} & \left(\left(\left|U_{\xi}\right|+\left(1+2 U^{2}+2 V^{2}\right) y_{\xi}\right) \chi_{S}+h \chi_{S^{c}}\right)(\xi) d \eta+y(\xi) \\
& =\xi
\end{aligned}
$$

or

$$
\begin{aligned}
& \int_{-\infty}^{\xi}\left(\left(\left|U_{\xi}\right|+\left(1+2 U^{2}+2 V^{2}\right) y_{\xi}\right) \chi_{y^{-1}(A)}+h \chi_{y^{-1}\left(A^{c}\right)}\right)(\xi) d \eta \\
& \quad+y(\xi)=\xi .
\end{aligned}
$$

Since $U_{\xi}=u_{x} \circ y y_{\xi}$ and $h=\left(u^{2}+u_{x}^{2}+v^{2}\right) \circ y y_{\xi}$ almost everywhere, after a change of variables in (78), we get

$$
\begin{aligned}
& \int_{-\infty}^{y(\xi)}\left(\left(\left|u_{x}\right|+\left(1+2 u^{2}+2 v^{2}\right)\right) \chi_{(A)}+\left(u^{2}+u_{x}^{2}+v^{2}\right) \chi_{\left(A^{c}\right)}\right) \\
& \quad \times(\xi) d \eta+y(\xi)=\xi .
\end{aligned}
$$

Hence, by definition, $y$ and $\bar{y}$ satisfy (72) and therefore they coincide; that is, $\bar{y}=y$. We have

$$
\begin{gathered}
(\bar{U}, \bar{V})=(\bar{u}, \bar{v}) \circ \bar{y}=(u, v) \circ y=(U, V), \\
\bar{h}=\bar{U}^{2} \bar{y}_{\xi}+\frac{\bar{U}_{\xi}^{2}}{\bar{y}_{\xi}}+\bar{V}^{2} \bar{y}_{\xi}=U^{2} y_{\xi}+\frac{U_{\xi}^{2}}{y_{\xi}}+V^{2} y_{\xi}=h
\end{gathered}
$$

almost everywhere. 
The bijective mapping $L$ allows us to transport the metric $\widetilde{d}_{R}$ on $\Gamma_{0}$ and the semigroup $\widetilde{S}_{t}$ from $\Gamma_{0}$ to $H^{1} \times W$. The metric $d_{H^{1}}$ on $H^{1} \times W$ and the semigroup $T_{t}$ on $H^{1}$ are given by

$$
d_{H^{1}}(z, \bar{z})=\widetilde{d}_{R}(L(z), L(\bar{z})), \quad T_{t}=\mathrm{M} \circ \widetilde{S}_{t} \circ L .
$$

Then a continuous semigroup of dissipative weak solutions for the two-component Camassa-Holm system is obtained as the following theorem shows.

Theorem 7. Let $z_{0}=\left(u_{0}, v_{0}\right) \in H^{1} \times W$ be given. If one denotes $t \rightarrow z(t)=T_{t}\left(z_{0}\right)$ to be the corresponding trajectory, then $z=(u, v)$ is a weak solution of the two-component CamassaHolm system (4), which constructs a continuous semigroup with respect to the metric $d_{H^{1}}$ on bounded sets of $H^{1} \times W$; that is, for any $M>0$ and any sequence $z_{n} \in H^{1} \times W$ such that $\left\|z_{n}\right\|_{H^{1}} \leq M$, one has that $\lim _{n \rightarrow \infty} d_{H^{1}}\left(z_{n}, z\right)=0$ implies $\lim _{n \rightarrow \infty} d_{H^{1}}\left(T_{t}\left(z_{n}\right), T_{t}(z)\right)=0$.

Proof. We denote $X_{0}=\mathrm{L}\left(z_{0}\right)$ and $X(t)=S_{t}\left(X_{0}\right)$. To prove $z=(u, v)$ is a weak solution of the original system (4), it suffices to show that, for all $\phi \in C^{\infty}\left(R^{+} \times R\right)$ with compact support,

$$
\begin{gathered}
\int_{R^{+} \times R}\left(-u \phi_{t}+u u_{x} \phi\right)(t, x) d x d t \\
=-\int_{R^{+} \times R}\left(P_{x} \phi\right)(t, x) d x d t, \\
\int_{R^{+} \times R}\left(-v \phi_{t}+u v_{x} \phi\right)(t, x) d x d t \\
=-\int_{R^{+} \times R}\left[(v+1) u_{x} \phi\right](t, x) d x d t,
\end{gathered}
$$

where $P_{x}$ is given by (4). On the one hand, since $y(t, \xi)$ is Lipschitz and invertible with respect to $\xi$ for almost all $t$, we then can use the change of variables $x=y(t, \xi)$ and obtain

$$
\begin{aligned}
\int_{R^{+} \times R}\left(-u \phi_{t}+u u_{x} \phi\right)(t, x) d x d t & \\
=\int_{R^{+} \times R}[- & -\left(U y_{\xi}\right)(t, \xi) \phi_{t}(t, y(t, \xi)) \\
& \left.+\left(U U_{\xi}\right)(t, \xi) \phi(t, y(t, \xi))\right] d \xi d t .
\end{aligned}
$$

We have $y_{\xi t}(t, \xi)=\chi_{\{t<\tau(\xi)\}}(\xi) U_{\xi}(t, \xi)$ and then $y_{\xi t}(t, \xi)=$ $U_{\xi}(t, \xi)$ as $U_{\xi}(t, \xi)=0$ for $t \geq \tau(\xi)$. By using the identity $y_{t}=U$, and since $y_{\xi}(t, \xi)=0$ for $t \geq \tau(\xi)$, it then follows from (20) that

$$
\begin{gathered}
\int_{R^{+} \times R}\left[-U y_{\xi} \phi_{t}(t, y)+U U_{\xi} \phi(t, y)\right] d \xi d t \\
=\frac{1}{4} \cdot \int_{R^{+} \times\{t<\tau(\xi)\} \times\left\{t<\tau\left(\xi^{\prime}\right)\right\}}\left\{\operatorname{sgn}\left(\xi-\xi^{\prime}\right) e^{-\left|y(\xi)-y\left(\xi^{\prime}\right)\right|}\right. \\
\left.\times\left[h+\left(U^{2}+2 V\right) y_{\xi}\right]\right\}\left(\xi^{\prime}\right) \\
\cdot \phi(t, y(\xi)) y_{\xi}(\xi) d \xi^{\prime} d \xi d t .
\end{gathered}
$$

On the other hand, using the change of variables $x=y(t, \xi)$ and $x^{\prime}=y\left(t, \xi^{\prime}\right)$, and since $y$ is increasing with respect to $\xi$, we have

$$
\begin{aligned}
&-\int_{R^{+} \times R}\left(P_{x} \phi\right)(t, x) d x d t \\
&=\frac{1}{2} \cdot \int_{R^{+} \times R^{2}} {\left[\operatorname{sgn}\left(\xi-\xi^{\prime}\right) e^{-\left|y(\xi)-y\left(\xi^{\prime}\right)\right|}\right.} \\
&\left.\times\left(u^{2}+\frac{u_{x}^{2}}{2}+\frac{v^{2}}{2}+v\right)\right]\left(t, y\left(\xi^{\prime}\right)\right) \\
& \cdot \phi(t, y(\xi)) y_{\xi}\left(\xi^{\prime}\right) y_{\xi}(\xi) d \xi^{\prime} d \xi d t .
\end{aligned}
$$

We restrict the integration domain to $R^{+} \times\{t<\tau(\xi)\} \times\{t<$ $\left.\tau\left(\xi^{\prime}\right)\right\}$ again because $y_{\xi}(t, \xi)=0$ for $t \geq \tau(\xi)$. Then it follows from $(22 \mathrm{c})$ that

$$
\begin{aligned}
-\int_{R^{+} \times R}( & \left(P_{x} \phi\right)(t, x) d x d t \\
=\frac{1}{4} \cdot \int_{R^{+} \times\{t<\tau(\xi)\} \times\left\{t<\tau\left(\xi^{\prime}\right)\right\}}\{ & \\
& \\
\times & \operatorname{sgn}\left(\xi-\xi^{\prime}\right) e^{-\left|y(\xi)-y\left(\xi^{\prime}\right)\right|} \\
& \phi(t, y(\xi)) y_{\xi}(\xi) d \xi^{\prime} d \xi d t .
\end{aligned}
$$

By comparing (84) and (86), we know that

$$
\begin{gathered}
\int_{R^{+} \times R}\left[-U y_{\xi} \phi_{t}(t, y)+U U_{\xi} \phi(t, y)\right] d \xi d t \\
=-\int_{R^{+} \times R}\left(P_{x} \phi\right)(t, x) d x d t .
\end{gathered}
$$

Hence, the first identity in (82) holds. The second identity in (82) follows in the same way. Given a converging sequence $z_{n} \in H^{1} \times W$ such that $\left\|z_{n}\right\|_{H^{1}} \leq M$, we have, by the definition of $L$, that $X_{n}=L\left(z_{n}\right) \rightarrow X=L(z)$ in $\widetilde{d}_{R}$ and $L\left(z_{n}\right) \in B_{\bar{M}}$ for some $\bar{M}$, and $\widetilde{S}_{t}\left(X_{n}\right) \rightarrow \widetilde{S}_{t}(X)$ in $\widetilde{d}_{R}$ and therefore $T_{t}\left(z_{n}\right) \rightarrow$ $T_{t}(z)$ in $d_{H^{1}}$ from the definition of $\widetilde{S}_{t}$.

\section{Multipeakon Solutions of the Original System}

We derive a new system of ordinary differential equations for the multipeakon solutions which is well-posed even when collisions occur in this section, and the variables $(y, U, V, N, h)$ are used to characterize multipeakons in a way that avoids the problems related to blowing up.

Solutions of the two-component Camassa-Holm system may experience wave breaking in the sense that the solution develops singularities in finite time, while keeping the $H^{1}$ norm finite. Extending the solution beyond wave breaking 
imposes significant challenge as can be illustrated in the case of multipeakons given by

$$
u(t, x)=\sum_{i=1}^{n} p_{i}(t) e^{-\left|x-q_{i}(t)\right|},
$$

where $\left(p_{i}(t), q_{i}(t)\right)$ satisfy the explicit system of ordinary differential equations

$$
\begin{gathered}
\dot{p}_{i}=\sum_{j=1}^{n} p_{i} p_{j} \operatorname{sgn}\left(q_{i}-q_{j}\right) e^{-\left|q_{i}-q_{j}\right|}, \\
\dot{q}_{i}=\sum_{j=1}^{n} p_{j} e^{-\left|q_{i}-q_{j}\right|} .
\end{gathered}
$$

Peakons interact in a way similar to that of solitons of the $\mathrm{CH}$ equation, and wave breaking may appear when at least two of the $q_{i}$ coincide. Clearly, if the $q_{i}$ remain distinct, the system (89) allows for a global smooth solution. In the case where $p_{i}(0)$ has the same sign for all $i=1,2, \ldots n$, then $q_{i}(t)$ remain distinct, and (89) admits a unique global solution. In this case, the peakons are traveling in the same direction. However, when two peakons have opposite signs, collisions may occur, and if so, the system (89) blows up.

We consider initial data $\bar{z}=(\bar{u}, \bar{v})$ given by

$$
(\bar{u}, \bar{v})(x)=\left(\sum_{i=1}^{n} p_{i} e^{-\left|x-\xi_{i}\right|}, \sum_{i=1}^{n} r_{i} e^{-\left|x-\xi_{i}\right|}\right) .
$$

Without loss of generality, we assume that the $p_{i}$ and $r_{i}$ are all nonzero and that the $\xi_{i}$ are all distinct. From Theorem 7 we know that there exists a unique and global weak solution with initial data (90), and the aim is to characterize this solution explicitly. We consider the following characterization of multipeakons. The multipeakons are given as continuous solutions $u$ defined on intervals $\left[x_{i}, x_{i+1}\right]$ as the solutions of the Dirichlet problem

$$
u-u_{x x}=0, \quad u\left(x_{i}\right)=u_{i}, \quad u\left(x_{i+1}\right)=u_{i+1},
$$

where the variables $x_{i}$ denote the position of the peaks and the variables $u_{i}$ denote the values of $u$ at the peaks. In the following we will show that this property persists for dissipative solutions.

Let us define $\bar{X}=(\bar{y}, \bar{U}, \bar{V}, \bar{N}, \bar{h})$ as

$$
\begin{gathered}
\bar{y}(\xi)=\xi, \\
\bar{U}(\xi)=\bar{u}(\xi), \quad \bar{v}(\xi)=\bar{v}(\xi), \quad \bar{N}(\xi)=\bar{u}_{x}(\xi) \\
\bar{h}(\xi)=\bar{u}^{2}+\bar{u}_{x}^{2}+\bar{v}^{2},
\end{gathered}
$$

which is a representative of $z=(u, v)$ in Lagrangian equivalent system; that is, $\bar{X}=L(\bar{z})$.

Let $I=\cup_{i=0}^{n} I_{i}$ where $I_{i}$ denote the open interval $\left(\xi_{i}, \xi_{i+1}\right)$ with the conventions that $\xi_{0}=-\infty$ and $\xi_{n+1}=\infty$. For each interval $I_{i}$, we define $\tau_{i}=\inf \left\{\tau(\xi) \mid \xi \in\left(\xi_{i}, \xi_{i+1}\right)\right\}$ such that $\tau_{i}>0$ for all $i=1, \ldots, n$. By the linearity of the governing equations (20) and the bounds which hold on the solution $X$ and $P, P_{x}$, it is not hard to check that $y, U, V \in$ $C\left(\left[0, \tau_{i}\right], C^{2}\left(I_{i}\right)\right)$, while $h \in C\left(\left[0, \tau_{i}\right], C^{1}\left(I_{i}\right)\right)$.

Thus the existence of multipeakon solutions is given by the following theorem.

Theorem 8. For any given multipeakon initial data $\bar{z}(x)=$ $(\bar{u}, \bar{v})(x)=\left(\sum_{i=1}^{n} p_{i} e^{-\left|x-\xi_{i}\right|}, \sum_{i=1}^{n} r_{i} e^{-\left|x-\xi_{i}\right|}\right)$, let $(y, U, V, N, h)$ be the solution of system (20), (21) with initial data $(\bar{y}, \bar{U}, \bar{V}, \bar{N}, \bar{h})$ given by (92a), (92b), and (92c). Between adjacent peaks, if $x_{i}=y\left(t, \xi_{i}\right) \neq x_{i+1}=y\left(t, \xi_{i+1}\right)$, the solution $u(t, x)$ is twice differentiable with respect to the space variable, and one has

$$
\left(u-u_{x x}\right)(t, x)=0 \quad \text { for } x \in\left(x_{i}, x_{i+1}\right) \text {. }
$$

Proof. For a given time $t$, we consider two adjacent peaks $x_{i}=y\left(t, \xi_{i}\right)$ and $x_{i+1}=y\left(t, \xi_{i+1}\right)$. If $x_{i}=x_{i+1}$, then the two peaks have collided and, since $y_{\xi}$ is positive, we must have $y_{\xi}(t, \xi)=0$ for all $\xi \in I_{i}$. Hence, $t \geq \tau_{i}$, which conversely implies that $t<\tau_{i}$ when $x_{i}(t)<x_{i+1}(t)$. There exists $\xi \in I_{i}$ such that $x=y(t, \xi)$ for any $x \in$ $\left(x_{i}(t), x_{i+1}(t)\right)$. Since $\xi \in I_{i}$ and $t<\tau_{i}$, we have $y_{\xi}(t, \xi) \neq 0$. It follows from the implicit function theorem that $y(t, \cdot)$ is invertible in a neighborhood of $\xi$ and its inverse is $C^{2}$, and therefore $u\left(t, x^{\prime}\right)=U\left(t, y^{-1}\left(t, x^{\prime}\right)\right)$ are $C^{2}$ with respect to the spatial variable and the quantity $\left(u-u_{x x}\right)(t, x)$ is defined in the classical sense.

We now prove that $\left(u-u_{x x}\right)(t, x)=0$ for $x \in\left(x_{i}, x_{i+1}\right)$. Assuming that $y_{\xi}(t, \xi) \neq 0$, we have

$$
u_{x} \circ y=N, \quad u_{x x} \circ y=\frac{N_{\xi}}{y_{\xi}}=\frac{\left(U_{\xi \xi} y_{\xi}-y_{\xi \xi} U_{\xi}\right)}{y_{\xi}^{3}} \text {, }
$$

and therefore

$$
\left(u-u_{x x}\right) \circ y=\frac{\left(U y_{\xi}^{3}-U_{\xi \xi} y_{\xi}+y_{\xi \xi} U_{\xi}\right)}{y_{\xi}^{3}} .
$$

We set

$$
M=U y_{\xi}^{3}-U_{\xi \xi} y_{\xi}+y_{\xi \xi} U_{\xi}
$$

For a given $\xi \in I$ and $t<\tau_{i}$, differentiating (96) with respect to $t$, it then follows from (20) and (21) that

$$
\begin{aligned}
\frac{d M}{d t}= & 3 U y_{\xi}^{2} y_{\xi t}+U_{t} y_{\xi}^{3}-U_{\xi \xi t} y_{\xi} \\
& -U_{\xi \xi} y_{\xi t}+y_{\xi \xi t} U_{\xi}+y_{\xi \xi} U_{\xi t} \\
= & 2 U U_{\xi} y_{\xi}^{2}-\frac{h_{\xi} y_{\xi}}{2}+\frac{h y_{\xi \xi}}{2}-V_{\xi} y_{\xi}^{2}
\end{aligned}
$$

Differentiating (22c) with respect to $\xi$, we get

$$
\begin{aligned}
y_{\xi \xi} h+y_{\xi} h_{\xi}= & 2 y_{\xi} y_{\xi \xi} U^{2}+2 y_{\xi}^{2} U U_{\xi}+2 U_{\xi} U_{\xi \xi} \\
& +2 y_{\xi} y_{\xi \xi} V^{2}+2 y_{\xi}^{2} V V_{\xi} .
\end{aligned}
$$


We have, after inserting the value of $y_{\xi} h_{\xi}$ given by (98) into (97) and multiplying the equation by $y_{\xi}$, that

$$
y_{\xi} \cdot \frac{d M}{d t}=U U_{\xi} y_{\xi}^{3}+U_{\xi}^{2} y_{\xi \xi}-U_{\xi} U_{\xi \xi} y_{\xi}
$$

Since $y_{\xi t}=U_{\xi}$, it follows from (22c) that

$$
y_{\xi} \cdot \frac{d M}{d t}=y_{\xi t} \cdot M
$$

For any $\xi \in I$, as $\bar{u}$ is multipeakon initial data, we have $M(0, \xi)=\left(\bar{u}-\bar{u}_{x x}\right) \cdot y_{\xi}^{3}=0$. It thus follows from Gronwall's lemma that $M(t, \xi)=0$ and therefore $\left(u-u_{x x}\right)(t, \xi)=0$ for $t \in\left[0, \tau_{i}\right]$.

Thus, the system of ordinary differential equations that the dissipative multipeakon solutions satisfy can be derived based on the fact that we have known that the multipeakon structure is preserved by the semigroup of dissipative solutions.

Let us define

$$
H_{i}=\int_{\xi_{i}}^{\xi_{i+1}} h(\xi) d \xi
$$

For each $i=1,2, \ldots, n$, by using (20), we obtain the following system of O.D.E.; namely,

$$
\begin{gathered}
\frac{d y_{i}}{d t}=u_{i}, \\
\frac{d u_{i}}{d t}=-P_{x i}, \\
\frac{d v_{i}}{d t}=-\left(v_{i}+1\right) u_{x i}, \\
\frac{d u_{x i}}{d t}=u_{i}^{2}-\frac{u_{x i}^{2}}{2}+\frac{v_{i}^{2}}{2}+v_{i}-P_{i}, \\
\frac{d H_{i}}{d t}=u_{i+1}^{3}-u_{i}^{3}-2 u_{i} P_{i}+2 u_{i+1} P_{i+1},
\end{gathered}
$$

where $\left(y_{i}, u_{i}, v_{i}, u_{x i} H_{i}\right)=(y, U, V, N, H)\left(t, \xi_{i}\right), P_{i}=P\left(t, \xi_{i}\right)$, and $P_{x i}=P_{x}\left(t, \xi_{i}\right)$. We have

$$
P_{i}=\frac{1}{4} \cdot \int_{t<\tau(\xi)} e^{-\left|y_{i}-y\left(\xi^{\prime}\right)\right|}\left[h+\left(U^{2}+2 V\right) y_{\xi}\right]\left(\xi^{\prime}\right) d \xi^{\prime}
$$

Let us denote $B=\left\{\xi^{\prime} \in R \mid y_{\xi}\left(t, \xi^{\prime}\right)>0\right\}$ and then we get

$$
\begin{aligned}
P_{i} & =\frac{1}{2} \cdot \int_{B} e^{-\left|y_{i}-y\left(\xi^{\prime}\right)\right|}\left(u^{2}+\frac{u_{x}^{2}}{2}+\frac{v^{2}}{2}+v\right) \circ y\left(\xi^{\prime}\right) y_{\xi}\left(\xi^{\prime}\right) d \xi^{\prime} \\
& =\frac{1}{2} \cdot \int_{y(B)} e^{-\left|y_{i}-x\right|}\left(u^{2}+\frac{u_{x}^{2}}{2}+\frac{v^{2}}{2}+v\right)(x) d x \\
& =\frac{1}{2} \cdot \int_{R} e^{-\left|y_{i}-x\right|}\left(u^{2}+\frac{u_{x}^{2}}{2}+\frac{v^{2}}{2}+v\right) d x,
\end{aligned}
$$

where we have used the fact that $h=\left(u^{2}+u_{x}^{2}+v^{2}\right) \circ y y_{\xi}$ on $B$ and $t<\tau\left(\xi^{\prime}\right)$ if and only if $y_{\xi}\left(t, \xi^{\prime}\right)>0$ and the domain of integration in (103) has been extended to the whole axis as $y_{\xi}(\xi)=0$ on $B^{c}$ which implies that meas $\left(y\left(B^{c}\right)\right)=\int_{y\left(B^{c}\right)} d x=$ $\int_{B^{c}} y_{\xi}(\xi) d \xi=0$. Similarly, we can get that

$$
P_{i, x}=-\frac{1}{2} \cdot \int_{R} \operatorname{sgn}\left(y_{i}-x\right) e^{-\left|y_{i}-x\right|}\left(u^{2}+\frac{u_{x}^{2}}{2}+\frac{v^{2}}{2}+v\right) d x .
$$

For $x \in\left[y_{i}, y_{i+1}\right], i=1,2, \ldots, n-1$, we write $z=(u, v)$ as

$$
z(x)=(u, v)(x)=\left(A_{i} e^{x}+B_{i} e^{-x}, C_{i} e^{x}+D_{i} e^{-x}\right)
$$

The constants $A_{i}, B_{i}, C_{i}$, and $D_{i}$ depend on $u_{i}, u_{i+1}, v_{i}, v_{i+1}, y_{i}$, and $y_{i+1}$ and read

$$
\begin{aligned}
A_{i} & =\frac{e^{-\bar{y}_{i}}}{2}\left[\frac{\bar{u}_{i}}{\cosh \left(\delta y_{i}\right)}+\frac{\delta u_{i}}{\sinh \left(\delta y_{i}\right)}\right], \\
B_{i} & =\frac{e^{\bar{y}_{i}}}{2}\left[\frac{\bar{u}_{i}}{\cosh \left(\delta y_{i}\right)}-\frac{\delta u_{i}}{\sinh \left(\delta y_{i}\right)}\right], \\
C_{i} & =\frac{e^{-\bar{y}_{i}}}{2}\left[\frac{\bar{v}_{i}}{\cosh \left(\delta y_{i}\right)}+\frac{\delta v_{i}}{\sinh \left(\delta y_{i}\right)}\right], \\
D_{i} & =\frac{e^{\bar{y}_{i}}}{2}\left[\frac{\bar{v}_{i}}{\cosh \left(\delta y_{i}\right)}-\frac{\delta v_{i}}{\sinh \left(\delta y_{i}\right)}\right],
\end{aligned}
$$

where

$$
\begin{array}{ll}
\bar{y}_{i}=\frac{1}{2} \cdot\left(y_{i}+y_{i+1}\right), & \delta y_{i}=\frac{1}{2} \cdot\left(y_{i}-y_{i+1}\right), \\
\bar{u}_{i}=\frac{1}{2} \cdot\left(u_{i}+u_{i+1}\right), & \delta u_{i}=\frac{1}{2} \cdot\left(u_{i}-u_{i+1}\right), \\
\bar{v}_{i}=\frac{1}{2} \cdot\left(v_{i}+v_{i+1}\right), & \delta v_{i}=\frac{1}{2} \cdot\left(v_{i}-v_{i+1}\right) .
\end{array}
$$

The constants $A_{i}, B_{i}, C_{i}$, and $D_{i}$ uniquely determine $z=$ $(u, v)$ on the interval $\left[y_{i}, y_{i+1}\right]$. We now turn to the computation of $P_{i}$ given by (106). Let us write $z=(u, v)$ as

$$
\begin{aligned}
z(t, x)= & (u(t, x), v(t, x)) \\
= & \left(\sum_{j=0}^{n}\left(A_{j} e^{x}+B_{j} e^{-x}\right) \chi_{\left(y_{j}, y_{j+1}\right)}(x),\right. \\
& \left.\sum_{j=0}^{n}\left(C_{j} e^{x}+D_{j} e^{-x}\right) \chi_{\left(y_{j}, y_{j+1}\right)}(x)\right) .
\end{aligned}
$$


We have set $y_{0}=-\infty, y_{n+1}=\infty, u_{0}=u_{n+1}=0, v_{0}=$ $v_{n+1}=0, A_{0}=u_{1} e^{-y_{1}}, B_{0}=0, A_{n}=0, B_{n}=u_{n} e^{y_{n}}$, and $C_{0}=v_{1} e^{-y_{1}}, D_{0}=0, C_{n}=0, D_{n}=v_{n} e^{y_{n}}$. We have

$$
\begin{aligned}
& u^{2}+ \frac{1}{2} u_{x}^{2}+\frac{1}{2} v^{2}+v \\
&=\sum_{j=0}^{n}\left(\frac{3}{2} A_{j}^{2} e^{2 x}+A_{j} B_{j}+\frac{3}{2} B_{j}^{2} e^{-2 x}+\frac{1}{2} C_{j}^{2} e^{2 x}\right. \\
&\left.\quad+C_{j} D_{j}+\frac{1}{2} D_{j}^{2} e^{-2 x}+C_{j} e^{x}+D_{j} e^{-x}\right) \chi_{\left(y_{j}, y_{j+1}\right)}
\end{aligned}
$$

Let

$$
k_{i j}= \begin{cases}1 & \text { if } i>j \\ -1 & \text { if } i \leq j .\end{cases}
$$

Inserting (110) into (106), we obtain

$$
\begin{aligned}
& P_{i}=\frac{1}{2} \sum_{j=0}^{n} \int_{y_{j}}^{y_{j+1}} e^{-k_{i j}\left(y_{i}-x\right)} \\
& \times\left(\frac{3}{2} A_{j}^{2} e^{2 x}+A_{j} B_{j}+\frac{3}{2} B_{j}^{2} e^{-2 x}\right. \\
& \quad+\frac{1}{2} C_{j}^{2} e^{2 x}+C_{j} D_{j}+\frac{1}{2} D_{j}^{2} e^{-2 x} \\
&\left.+C_{j} e^{x}+D_{j} e^{-x}\right) d x .
\end{aligned}
$$

From (108) and (110), we get

$$
\begin{aligned}
A_{j}^{2}= & \frac{e^{-2 \bar{y}_{j}}}{\sinh ^{2}\left(2 \delta y_{j}\right)} \\
& \times\left[\bar{u}_{j}^{2} \sinh ^{2}\left(\delta y_{j}\right)+2 \bar{u}_{j} \delta u_{j} \sinh \left(\delta y_{j}\right) \cosh \left(\delta y_{j}\right)\right. \\
& \left.\quad+\delta u_{j}^{2} \cosh ^{2}\left(\delta y_{j}\right)\right] \\
= & \frac{e^{-2 \bar{y}_{j}}}{2 \sinh \left(2 \delta y_{j}\right)} \\
& \times\left[\bar{u}_{j}^{2} \tanh \left(\delta y_{j}\right)+2 \bar{u}_{j} \delta u_{j}+\delta u_{j}^{2} \operatorname{coth}\left(\delta y_{j}\right)\right], \\
B_{j}^{2}= & \frac{e^{2 \bar{y}_{j}}}{2 \sinh \left(2 \delta y_{j}\right)} \\
& \times\left[\bar{u}_{j}^{2} \tanh \left(\delta y_{j}\right)-2 \bar{u}_{j} \delta u_{j}+\delta u_{j}^{2} \operatorname{coth}\left(\delta y_{j}\right)\right], \\
A_{j} B_{j}= & \frac{1}{4 \sinh \left(2 \delta y_{j}\right)}\left[2 \bar{u}_{j}^{2} \tanh \left(\delta y_{j}\right)-2 \delta u_{j}^{2} \operatorname{coth}\left(\delta y_{j}\right)\right], \\
C_{j}^{2}= & \frac{e^{-2 \bar{y}_{j}}}{2 \sinh \left(2 \delta y_{j}\right)} \\
& \times\left[\bar{v}_{j}^{2} \tanh \left(\delta y_{j}\right)+2 \bar{v}_{j} \delta v_{j}+\delta v_{j}^{2} \operatorname{coth}\left(\delta y_{j}\right)\right],
\end{aligned}
$$

$$
\begin{aligned}
D_{j}^{2}= & \frac{e^{2 \bar{y}_{j}}}{2 \sinh \left(2 \delta y_{j}\right)} \\
& \times\left[\bar{v}_{j}^{2} \tanh \left(\delta y_{j}\right)-2 \bar{v}_{j} \delta v_{j}+\delta v_{j}^{2} \operatorname{coth}\left(\delta y_{j}\right)\right], \\
C_{j} D_{j}= & \frac{1}{4 \sinh \left(2 \delta y_{j}\right)}\left[2 \bar{v}_{j}^{2} \tanh \left(\delta y_{j}\right)-2 \delta v_{j}^{2} \operatorname{coth}\left(\delta y_{j}\right)\right] .
\end{aligned}
$$

It then follows from (113) that

$$
\begin{aligned}
& \int_{y_{j}}^{y_{j+1}} e^{-k_{i j}\left(y_{i}-x\right)} A_{j}^{2} e^{2 x} d x \\
& =\frac{e^{-k_{i j} y_{i}} \cdot e^{k_{i j} \bar{y}_{j}}}{\left(2+k_{i j}\right) \sinh \left(2 \delta y_{j}\right)} \sinh \left(\left(2+k_{i j}\right) \delta y_{j}\right) \\
& \times\left[\bar{u}_{j}^{2} \tanh \left(\delta y_{j}\right)+2 \bar{u}_{j} \delta u_{j}+\delta u_{j}^{2} \operatorname{coth}\left(\delta y_{j}\right)\right], \\
& \int_{y_{j}}^{y_{j+1}} e^{-k_{i j}\left(y_{i}-x\right)} B_{j}^{2} e^{-2 x} d x \\
& =\frac{e^{-k_{i j} y_{i}} \cdot e^{k_{i j} \bar{y}_{j}}}{\left(k_{i j}-2\right) \sinh \left(2 \delta y_{j}\right)} \sinh \left(\left(k_{i j}-2\right) \delta y_{j}\right) \\
& \times\left[\bar{u}_{j}^{2} \tanh \left(\delta y_{j}\right)-2 \bar{u}_{j} \delta u_{j}+\delta u_{j}^{2} \operatorname{coth}\left(\delta y_{j}\right)\right], \\
& \int_{y_{j}}^{y_{j+1}} e^{-k_{i j}\left(y_{i}-x\right)} C_{j}^{2} e^{2 x} d x \\
& =\frac{e^{-k_{i j} y_{i}} \cdot e^{k_{i j} \bar{y}_{j}}}{\left(2+k_{i j}\right) \sinh \left(2 \delta y_{j}\right)} \sinh \left(\left(2+k_{i j}\right) \delta y_{j}\right) \\
& \times\left[\bar{v}_{j}^{2} \tanh \left(\delta y_{j}\right)+2 \bar{v}_{j} \delta v_{j}+\delta v_{j}^{2} \operatorname{coth}\left(\delta y_{j}\right)\right], \\
& \int_{y_{j}}^{y_{j+1}} e^{-k_{i j}\left(y_{i}-x\right)} D_{j}^{2} e^{-2 x} d x \\
& =\frac{e^{-k_{i j} y_{i}} \cdot e^{k_{i j} \bar{y}_{j}}}{\left(k_{i j}-2\right) \sinh \left(2 \delta y_{j}\right)} \sinh \left(\left(k_{i j}-2\right) \delta y_{j}\right) \\
& \times\left[\bar{v}_{j}^{2} \tanh \left(\delta y_{j}\right)-2 \bar{v}_{j} \delta v_{j}+\delta v_{j}^{2} \operatorname{coth}\left(\delta y_{j}\right)\right] . \\
& \int_{y_{j}}^{y_{j+1}} e^{-k_{i j}\left(y_{i}-x\right)} A_{j} B_{j} d x \\
& =\frac{e^{-k_{i j} y_{i}} \cdot e^{k_{i j} \bar{y}_{j}}}{2 \sinh \left(2 \delta y_{j}\right)} \sinh \left(\delta y_{j}\right) \\
& \times\left[2 \bar{u}_{j}^{2} \tanh \left(\delta y_{j}\right)-2 \delta u_{j}^{2} \operatorname{coth}\left(\delta y_{j}\right)\right], \\
& \int_{y_{j}}^{y_{j+1}} e^{-k_{i j}\left(y_{i}-x\right)} C_{j} D_{j} d x
\end{aligned}
$$




$$
\begin{aligned}
= & \frac{e^{-k_{i j} y_{i}} \cdot e^{k_{i j} \bar{y}_{j}}}{2 \sinh \left(2 \delta y_{j}\right)} \sinh \left(\delta y_{j}\right) \\
& \times\left[2 \bar{v}_{j}^{2} \tanh \left(\delta y_{j}\right)-2 \delta v_{j}^{2} \operatorname{coth}\left(\delta y_{j}\right)\right] .
\end{aligned}
$$

Therefore, the above formulas in (114) imply that

$$
\begin{aligned}
& P_{i}=\sum_{j=0}^{n} \frac{e^{-k_{i j} y_{i}} \cdot e^{k_{i j} \bar{y}_{j}}}{4 \cosh \left(\delta y_{j}\right)}\left\{\left[\bar{u}_{j}^{2} \tanh \left(\delta y_{j}\right)+\delta u_{j}^{2} \operatorname{coth}\left(\delta y_{j}\right)\right]\right. \\
& \cdot 2 \cosh ^{2}\left(\delta y_{j}\right)+4 k_{i j} \bar{u}_{j} \delta u_{j} \sinh ^{2}\left(\delta y_{j}\right) \\
&+ 2 \bar{u}_{j}^{2} \tanh \left(\delta y_{j}\right)+\frac{2}{3} \sinh ^{2}\left(\delta y_{j}\right) \\
& \cdot {\left[\bar{v}_{j}^{2} \tanh \left(\delta y_{j}\right)+\delta v_{j}^{2} \operatorname{coth}\left(\delta y_{j}\right)\right] } \\
&+\frac{4}{3} k_{i j} \bar{v}_{j} \delta v_{j} \sinh { }^{2}\left(\delta y_{j}\right)+2 \bar{v}_{j}^{2} \\
& \cdot \tanh (\left.\delta y_{j}\right)+2\left[\bar{v}_{j}-k_{i j} \delta v_{j} \operatorname{coth}\left(\delta y_{j}\right)\right] \\
& \cdot \delta y_{j}+ {\left[\bar{v}_{j} \sinh \left(\delta y_{j}\right)+k_{i j} \delta v_{j} \cosh \left(\delta y_{j}\right)\right] } \\
&\left.\cdot 2 \cosh \left(\delta y_{j}\right)\right\},
\end{aligned}
$$

which can also be written in the following form:

$$
P_{i}=\sum_{j=0}^{n} P_{i j}
$$

with

$$
P_{i j}=\left\{\begin{array}{l}
\frac{1}{4} u_{1}^{2} e^{y_{1}-y_{i}}+\frac{1}{12} v_{1}^{2} e^{y_{1}-y_{i}}+\frac{1}{4} v_{1} e^{y_{1}-y_{i}}, \\
\quad \text { for } j=0, \\
\frac{e^{-k_{i j} y_{i}} \cdot e^{k_{i j} \bar{y}_{j}}}{4 \cosh \left(\delta y_{j}\right)}\left\{\left[\bar{u}_{j}^{2} \tanh \left(\delta y_{j}\right)+\delta u_{j}^{2} \operatorname{coth}\left(\delta y_{j}\right)\right]\right. \\
\cdot 2 \cosh ^{2}\left(\delta y_{j}\right)+4 k_{i j} \bar{u}_{j} \delta u_{j} \sinh ^{2}\left(\delta y_{j}\right) \\
+2 \bar{u}_{j}^{2} \tanh \left(\delta y_{j}\right)+\frac{2}{3} \sinh ^{2}\left(\delta y_{j}\right) \\
\cdot\left[\bar{v}_{j}^{2} \tanh \left(\delta y_{j}\right)+\delta v_{j}^{2} \operatorname{coth}\left(\delta y_{j}\right)\right] \\
+\frac{4}{3} k_{i j} \bar{v}_{j} \delta v_{j} \sinh \left(\delta y_{j}\right)+2 \bar{v}_{j}^{2} \tanh \left(\delta y_{j}\right) \\
+2\left[\bar{v}_{j}-k_{i j} \delta v_{j} \operatorname{coth}\left(\delta y_{j}\right)\right] \\
\cdot \delta y_{j}+\left[\bar{v}_{j} \sinh \left(\delta y_{j}\right)+k_{i j} \delta v_{j} \cosh \left(\delta y_{j}\right)\right] \\
\left.\cdot 2 \cosh \left(\delta y_{j}\right)\right\}, \\
\quad \text { for } j=1, \ldots, n-1, \\
\frac{1}{4} u_{n}^{2} e^{y_{i}-y_{n}}+\frac{1}{12} v_{n}^{2} e^{y_{i}-y_{n}}+\frac{1}{4} v_{n} e^{y_{i}-y_{n}}, \\
\text { for } j=n .
\end{array}\right.
$$

We compute $P_{i x}$ in the same way and obtain

$$
P_{i, x}=-\sum_{j=0}^{n} k_{i j} P_{i j} .
$$

Now we can summarize the result as follows.

Theorem 9. Let $\bar{y}_{i}=\xi_{i},\left(\bar{u}_{i}, \bar{v}_{i}, \bar{u}_{x i}\right)=\left(\bar{u}, \bar{v}, \bar{u}_{x}\right)\left(\xi_{i}\right)$, and $\bar{H}_{i}=\int_{-\infty}^{\xi_{i}}\left(\bar{u}^{2}+\bar{u}_{x}^{2}+\bar{v}^{2}\right) d x$ for $i=1, \ldots, n$ with multipeakon initial data $\bar{z}=(\bar{u}, \bar{v})$ given by (90). Then, with initial data $\left(\bar{y}_{i}, \bar{u}_{i}, \bar{v}_{i}, \bar{u}_{x i}, \bar{H}_{i}\right)$, there exists a global solution $\left(y_{i}, u_{i}, v_{i}, u_{x i}, H_{i}\right)$ of (105), (116), and (118). For each time $t, u(t, x)$ is defined as the solution of the Dirichlet problem $u-u_{x x}=0$ with boundary conditions $u\left(t, y_{i}(t)\right)=u_{i}(t)$, $u\left(t, y_{i+1}(t)\right)=u_{i+1}(t)$ on each interval $\left[y_{i}(t), y_{i+1}(t)\right]$. Thus $z=$ $(u, v)$ is a dissipative solution of the two-component CamassaHolm system, which is the dissipative multipeakon solution.

In the following, we give the examples with cases $n=1,2$ with collision and without collision.

\section{Numerical Examples}

Two examples are considered here to illustrate the property of the system solution. The first is when $n=1$, and the second is when $n=2$.

(i) Let $n=1$. From (116) and (118), we can compute that $P_{1}=\sum_{j=0,1} P_{1 j}=u_{1}^{2} / 2+v_{1}^{2} / 6+v_{1} / 2$ and $P_{1, x}=$ $-\sum_{j=0,1} k_{1 j} P_{1 j}=0$. Thus from (102), we obtain that $u_{1}=c$ and $y_{1}=c t+a$ with $c, a$ some constants. There is no collision and we find the one peakon $u(t, x)=$ $c e^{-|x-c t-a|}$.

(ii) Let $n=2$. We consider the case of an antisymmetric pair of peakons when the two peakons collide. We take the initial conditions as

$$
\begin{gathered}
y_{2}(0)=-y_{1}(0)=\bar{y}, \quad u_{2}(0)=-u_{1}(0)=\bar{u}, \\
v_{2}(0)=-v_{1}(0)=\bar{v}, \quad \delta H_{1}(0)=E^{2}
\end{gathered}
$$

for some strictly positive constants $\bar{y}, \bar{u}, \bar{v}$ and $E$ (the initial total energy of the system). We denote by $\tau=\tau_{1}$ the time of collision. For $t<\tau$, the solution is identical to the conservative case. After collision, for $t \geq \tau$, the solution remains antisymmetric. Let us assume this for the moment and write

$$
\begin{gathered}
y=y_{2}=-y_{1}, \quad u=u_{2}=-u_{1}, \quad h=\delta H_{1}, \\
P=P_{1}=P_{2}, \quad P_{x}=-P_{1, x}=P_{2, x} .
\end{gathered}
$$


Using (116) and (118) we can compute $P$ and $P_{x}$ and obtain after some calculations that

$$
\begin{aligned}
P= & \sum_{j=0,2} P_{1, j}=\frac{1}{12} \cdot\left(3 u^{2}+v^{2}\right)\left(e^{-2 y}+1\right) \\
& +\frac{1}{4} \cdot v\left(e^{-2 y}-1\right), \\
P_{x}= & -\sum_{j=0,2} k_{1, j} P_{1, j}=\frac{1}{12} \cdot\left(3 u^{2}+v^{2}\right)\left(e^{-2 y}-1\right) \\
& +\frac{1}{4} \cdot v\left(e^{-2 y}+1\right) .
\end{aligned}
$$

Thus we are led to the following system of ordinary differential equations:

$$
\begin{gathered}
y_{t}=u, \\
u_{t}=\frac{1}{12} \cdot\left(3 u^{2}+v^{2}\right)\left(1-e^{-2 y}\right)-\frac{1}{4} \cdot v\left(1+e^{-2 y}\right), \\
h_{t}=\left(u^{3}+u v\right)\left(1-e^{-2 y}\right)-\frac{1}{3} \cdot u v^{2}\left(1+e^{-2 y}\right) .
\end{gathered}
$$

Note that this system holds before collision. The solution of (122) with the initial condition $y(\tau)=u(\tau)=v(\tau)=0$ is $y(t)=u(t)=v(t)=0$ and $h(t)=h(\tau)$. It means that the multipeakon solution remains identically equal to zero after the collision.

We can get from (116) that

$$
\begin{gathered}
P_{1,0}=\frac{1}{4} \cdot\left(u_{1}^{2}+\frac{1}{3} \cdot v_{1}^{2}+v_{1}\right), \\
P_{1,2}=\frac{1}{4} \cdot e^{y_{1}-y_{2}}\left(u_{2}^{2}+\frac{1}{3} \cdot v_{2}^{2}+v_{2}\right), \\
P_{2,0}=\frac{1}{4} \cdot e^{y_{1}-y_{2}}\left(u_{1}^{2}+\frac{1}{3} \cdot v_{1}^{2}+v_{1}\right), \\
P_{2,2}=\frac{1}{4} \cdot\left(u_{2}^{2}+\frac{1}{3} \cdot v_{2}^{2}+v_{2}\right) .
\end{gathered}
$$

Thus, for $t \geq \tau$, we have

$$
\begin{aligned}
P_{1}=P_{1,0}+P_{1,2}= & \frac{1}{4} \cdot\left(u_{1}^{2}+\frac{1}{3} \cdot v_{1}^{2}+v_{1}\right) \\
& +\frac{1}{4} \cdot e^{y_{1}-y_{2}}\left(u_{2}^{2}+\frac{1}{3} \cdot v_{2}^{2}+v_{2}\right), \\
P_{2}=P_{2,0}+P_{2,2}= & \frac{1}{4} \cdot e^{y_{1}-y_{2}}\left(u_{1}^{2}+\frac{1}{3} \cdot v_{1}^{2}+v_{1}\right) \\
& +\frac{1}{4} \cdot\left(u_{2}^{2}+\frac{1}{3} \cdot v_{2}^{2}+v_{2}\right), \\
P_{1, x}=-P_{1,0}+P_{1,2}= & -\frac{1}{4} \cdot\left(u_{1}^{2}+\frac{1}{3} \cdot v_{1}^{2}+v_{1}\right) \\
& +\frac{1}{4} \cdot e^{y_{1}-y_{2}}\left(u_{2}^{2}+\frac{1}{3} \cdot v_{2}^{2}+v_{2}\right), \\
P_{2, x}=-P_{2,0}+P_{2,2}= & -\frac{1}{4} \cdot e^{y_{1}-y_{2}}\left(u_{1}^{2}+\frac{1}{3} \cdot v_{1}^{2}+v_{1}\right) \\
& +\frac{1}{4} \cdot\left(u_{2}^{2}+\frac{1}{3} \cdot v_{2}^{2}+v_{2}\right),
\end{aligned}
$$

and therefore the system (102) rewrites

$$
\begin{gathered}
\frac{d y_{1}}{d t}=u_{1}, \quad \frac{d y_{2}}{d t}=u_{2}, \\
\frac{d u_{1}}{d t}=\frac{1}{4} \cdot\left(u_{1}^{2}+\frac{1}{3} \cdot v_{1}^{2}+v_{1}\right) \\
-\frac{1}{4} \cdot e^{y_{1}-y_{2}}\left(u_{2}^{2}+\frac{1}{3} \cdot v_{2}^{2}+v_{2}\right), \\
\frac{d u_{2}}{d t}=\frac{1}{4} \cdot e^{y_{1}-y_{2}}\left(u_{1}^{2}+\frac{1}{3} \cdot v_{1}^{2}+v_{1}\right) \\
-\frac{1}{4} \cdot\left(u_{2}^{2}+\frac{1}{3} \cdot v_{2}^{2}+v_{2}\right), \\
\frac{d H_{1}}{d t}=u_{2}^{3}-u_{1}^{3}-2 u_{1} P_{1}+2 u_{2} P_{2} .
\end{gathered}
$$

If we consider a more general case with two colliding peakons by using the Hamiltonian system before collision, we have

$$
\begin{aligned}
& y_{1}=\ln \left(\frac{c_{1}-c_{2}}{c_{1} e^{-c_{1}(t-\tau)}-c_{2} e^{-c_{2}(t-\tau)}}\right), \\
& y_{2}=\ln \left(\frac{c_{1} e^{c_{1}(t-\tau)}-c_{2} e^{c_{2}(t-\tau)}}{c_{1}-c_{2}}\right), \\
& u_{1}=\frac{c_{1}^{2}-c_{2}^{2} e^{\left(c_{1}-c_{2}\right)(t-\tau)}}{c_{1}-c_{2} e^{\left(c_{1}-c_{2}\right)(t-\tau)}} \\
& u_{2}=\frac{c_{2}^{2}-c_{1}^{2} e^{\left(c_{1}-c_{2}\right)(t-\tau)}}{c_{2}-c_{1} e^{\left(c_{1}-c_{2}\right)(t-\tau)}},
\end{aligned}
$$

where $c_{1}, c_{2}$ denote the speed of the peaks $y_{1}$ and $y_{2}$. At collision time, we have

$$
\begin{gathered}
y_{1}(\tau)=y_{2}(\tau)=0, \\
u_{1}(\tau)=u_{2}(\tau)=c_{1}+c_{2} .
\end{gathered}
$$

Thus, for the initial data given by (127), the solution of (125) is

$$
\begin{gathered}
u_{1}(t)=u_{2}(t)=c_{1}+c_{2}, \\
y_{1}=y_{2}=\left(c_{1}+c_{2}\right) t, \quad H_{1}(t)=H_{1}(\tau)
\end{gathered}
$$

and after the collision we obtain a single peakon traveling at speed $c_{1}+c_{2}$. Figures 1 and 2 represent the solution for $c_{1}=15$, $c_{2}=-5$, and $\tau=1$, respectively.

\section{Conclusion}

Considered in this paper is the dissipative property of the two-component Camassa-Holm system after wave breaking. By using a new approach, we obtain the global dissipative solutions of the two-component Camassa-Holm system and then the multipeakon dissipative solutions, a useful result for understanding the inevitable multipeakon phenomenon near wave breaking. 


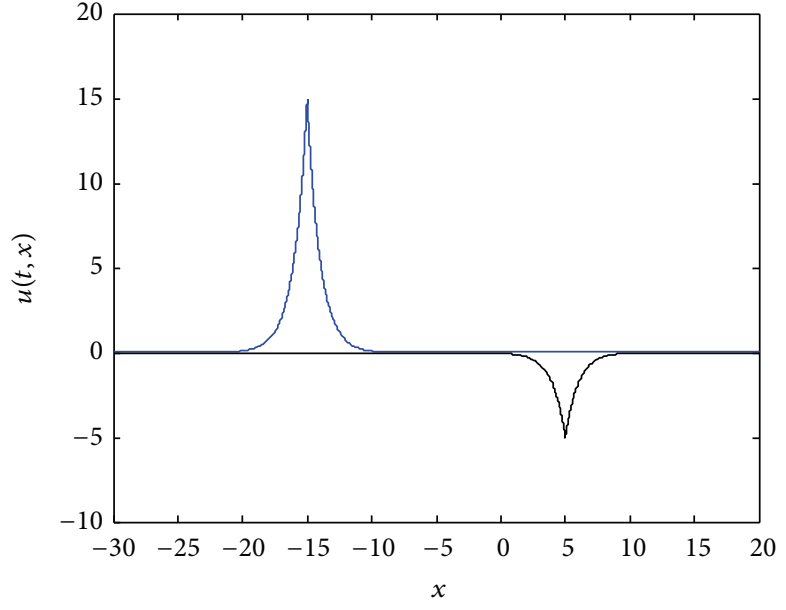

(a)

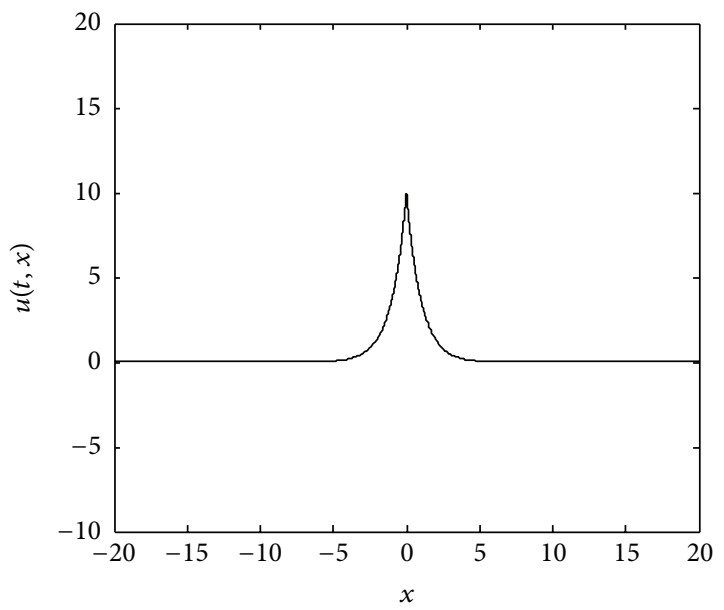

(b)

Figure 1: (a) Initial configuration. (b) The two peakons collide.

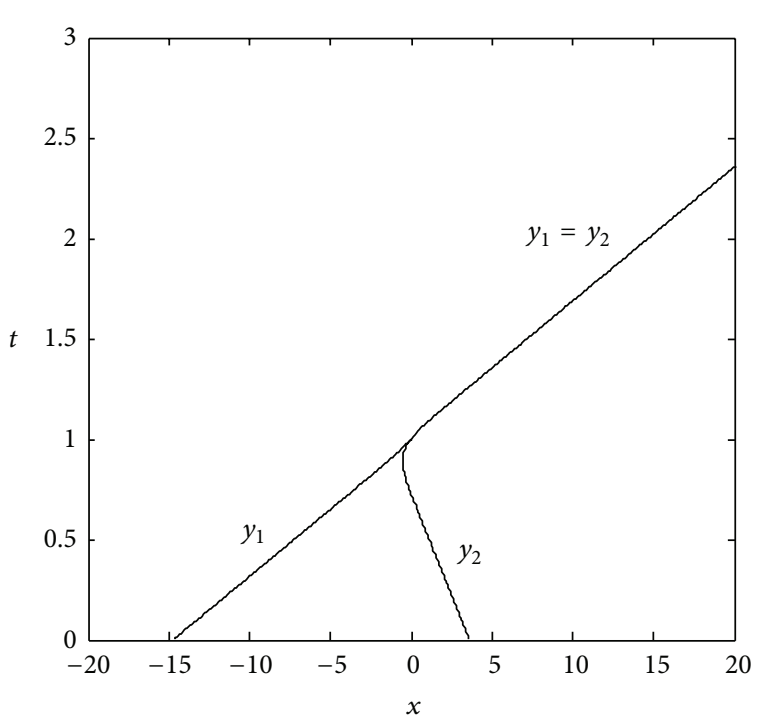

FIGURE 2: Position of the peakons.

\section{Conflict of Interests}

The authors declare that there is no conflict of interests regarding the publication of this paper.

\section{Acknowledgments}

This work was supported in part by the Major State Basic Research Development Program 973 (no. 2012CB215202), the National Natural Science Foundation of China (no. 61134001), and the Key Laboratory of Dependable Service Computing in Cyber Physical Society (Chongqing University), Ministry of Education.

\section{References}

[1] R. Camassa and D. Holm, "An integrable shallow water equation with peaked solitons," Physical Review Letters, vol. 71, no. 11, pp. 1661-1664, 1993.

[2] A. Constantin and J. Escher, "Wave breaking for nonlinear nonlocal shallow water equations," Acta Mathematica, vol. 181, no. 2, pp. 229-243, 1998.

[3] A. Constantin, "The Hamiltonian structure of the CamassaHolm equation," Expositiones Mathematicae, vol. 15, no. 1, pp. 53-85, 1997.

[4] A. Constantin, "On the scattering problem for the CamassaHolm equation," Proceedings of the the Royal Society of London Series A: Mathematical, Physical and Engineering Sciences, vol. 457, no. 2008, pp. 953-970, 2001.

[5] A. Constantin, "Global existence of solutions and breaking waves for a shallow water equation: a geometric approach," Annales de l'Institut Fourier, vol. 50, no. 2, pp. 321-362, 2000.

[6] A. Constantin and D. Lannes, "The hydrodynamical relevance of the Camassa-Holm and Degasperis-Procesi equations," Archive for Rational Mechanics and Analysis, vol. 192, no. 1, pp. 165-186, 2009.

[7] A. Bressan and A. Constantin, "Global conservative solutions of the Camassa-Holm equation," Archive for Rational Mechanics and Analysis, vol. 183, no. 2, pp. 215-239, 2007.

[8] H. Holden and X. Raynaud, "Global conservative solutions of the Camassa-Holm equation-a lagrangian point of view," Communications in Partial Differential Equations, vol. 32, no. 10, pp. 1511-1549, 2007.

[9] H. Holden and X. Raynaud, "Global conservative multipeakon solutions of the Camassa-Holm equation," Journal of Hyperbolic Differential Equations, vol. 4, no. 1, pp. 39-64, 2007.

[10] A. Bressan and A. Constantin, "Global dissipative solutions of the Camassa-Holm equation," Analysis and Applications, vol. 5, no. 1, pp. 1-27, 2007.

[11] H. Holden and X. Raynaud, "Dissipative solutions for the Camassa-Holm equation," Discrete and Continuous Dynamical Systems, vol. 24, no. 4, pp. 1047-1112, 2009.

[12] H. Holden and X. Raynaud, "Global dissipative multipeakon solutions of the Camassa-Holm equation," Communications in 
Partial Differential Equations, vol. 33, no. 11, pp. 2040-2063, 2008.

[13] A. Constantin and R. Ivanov, "On an integrable two-component Camassa-Holm shallow water system," Physics Letters A, vol. 372, no. 48, pp. 7129-7132, 2008.

[14] M. Chen, S.-Q. Liu, and Y. Zhang, "A two-component generalization of the Camassa-Holm equation and its solutions," Letters in Mathematical Physics, vol. 75, no. 1, pp. 1-15, 2006.

[15] G. Falqui, "On a Camassa-Holm type equation with two dependent variables," Journal of Physics A: Mathematical and General, vol. 39, no. 2, pp. 327-342, 2006.

[16] P. Olver and P. Rosenau, "Tri-Hamiltonian duality between solitons and solitary-wave solutions having compact support," Physical Review E, vol. 53, Article ID 1900, 1996.

[17] R. S. Johnson, "Camassa-Holm, Korteweg-de Vries and related models for water waves," Journal of Fluid Mechanics, vol. 455, pp. 63-82, 2002.

[18] G. Gui and Y. Liu, "On the Cauchy problem for the twocomponent Camassa-Holm system," Mathematische Zeitschrift, vol. 268, no. 1-2, pp. 45-66, 2011.

[19] J. Escher, O. Lechtenfeld, and Z. Y. Yin, "Well-posedness and blow-up phenomena for the 2-component Camassa-Holm equation," Discrete and Continuous Dynamical Systems, vol. 19, no. 3, pp. 493-513, 2007.

[20] Y. Wang and Y. Song, "Global conservative and multipeakon conservative solutions for the two-component Camassa-Holm system," Boundary Value Problems, vol. 2013, article 165, 2013.

[21] C. Guan and Z. Y. Yin, "Global existence and blow-up phenomena for an integrable two-component Camassa-Holm shallow water system," Journal of Differential Equations, vol. 248, no. 8, pp. 2003-2014, 2010.

[22] G. Gui and Y. Liu, "On the global existence and wave-breaking criteria for the two-component Camassa-Holm system," Journal of Functional Analysis, vol. 258, no. 12, pp. 4251-4278, 2010.

[23] C. Guan and Z. Yin, "Global weak solutions for a twocomponent Camassa-Holm shallow water system," Journal of Functional Analysis, vol. 260, no. 4, pp. 1132-1154, 2011.

[24] Y. Wang and Y. Song, "On the global existence of dissipative solutions for the modified coupled Camassa-Holm system," Soft Computing, vol. 17, no. 1, pp. 2007-2019, 2013.

[25] Z. Shen, Y. Wang, H. Karimi, and Y. Song, "On the multipeakon dissipative behavior of the modiied coupled Camassa-Holm model for shallow water system," Mathematical Problems in Engineering, vol. 2013, Article ID 107450, 11 pages, 2013. 


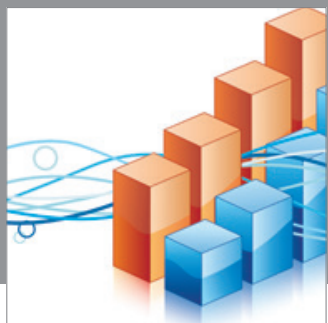

Advances in

Operations Research

mansans

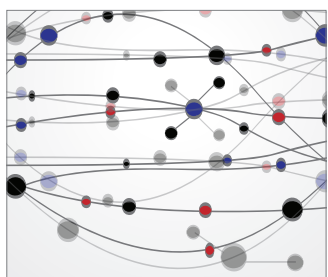

The Scientific World Journal
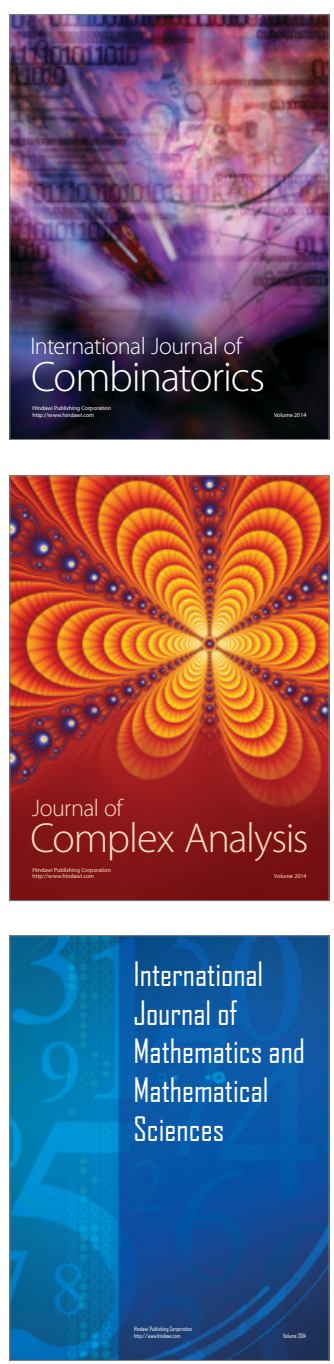
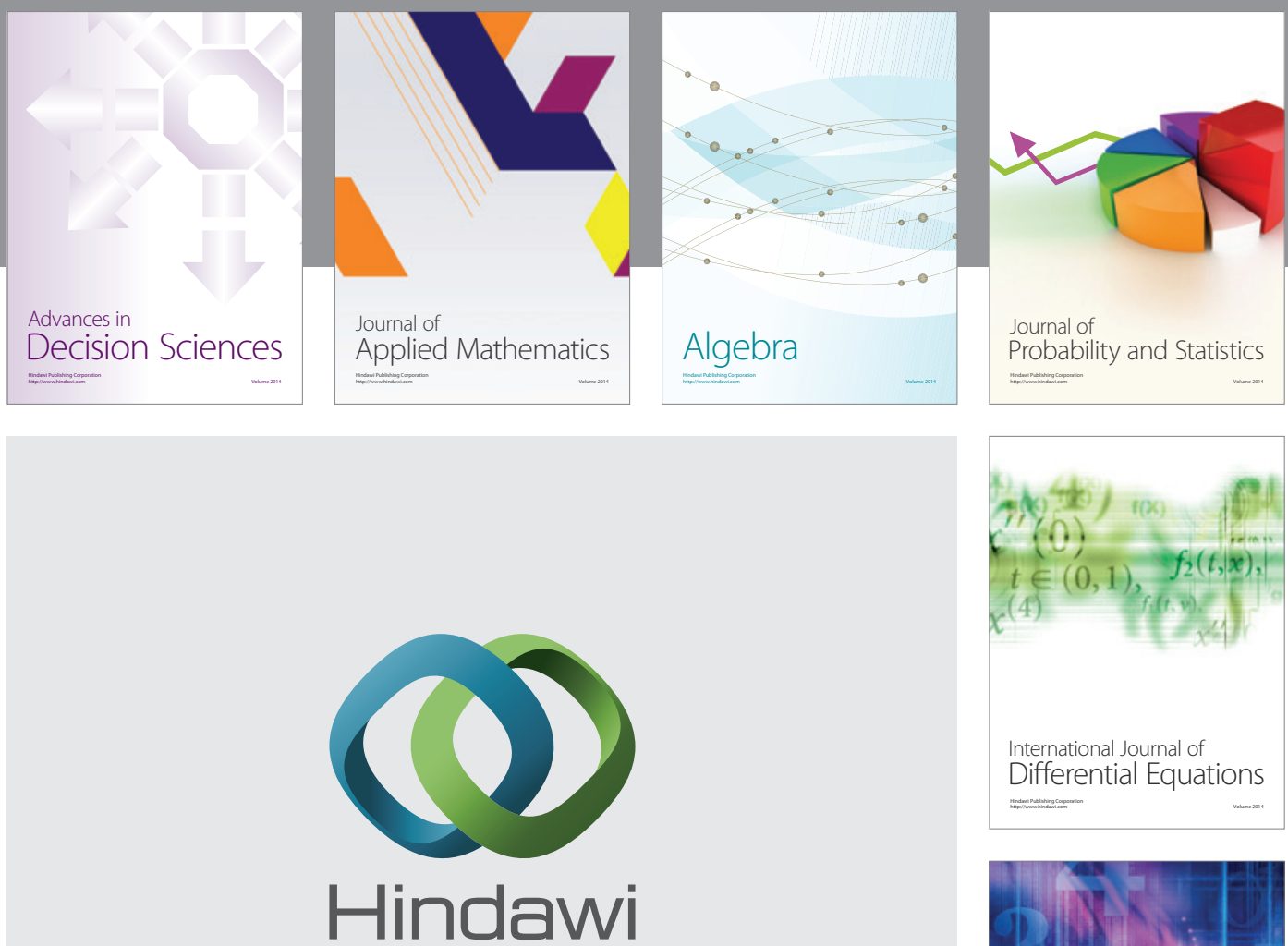

Submit your manuscripts at http://www.hindawi.com
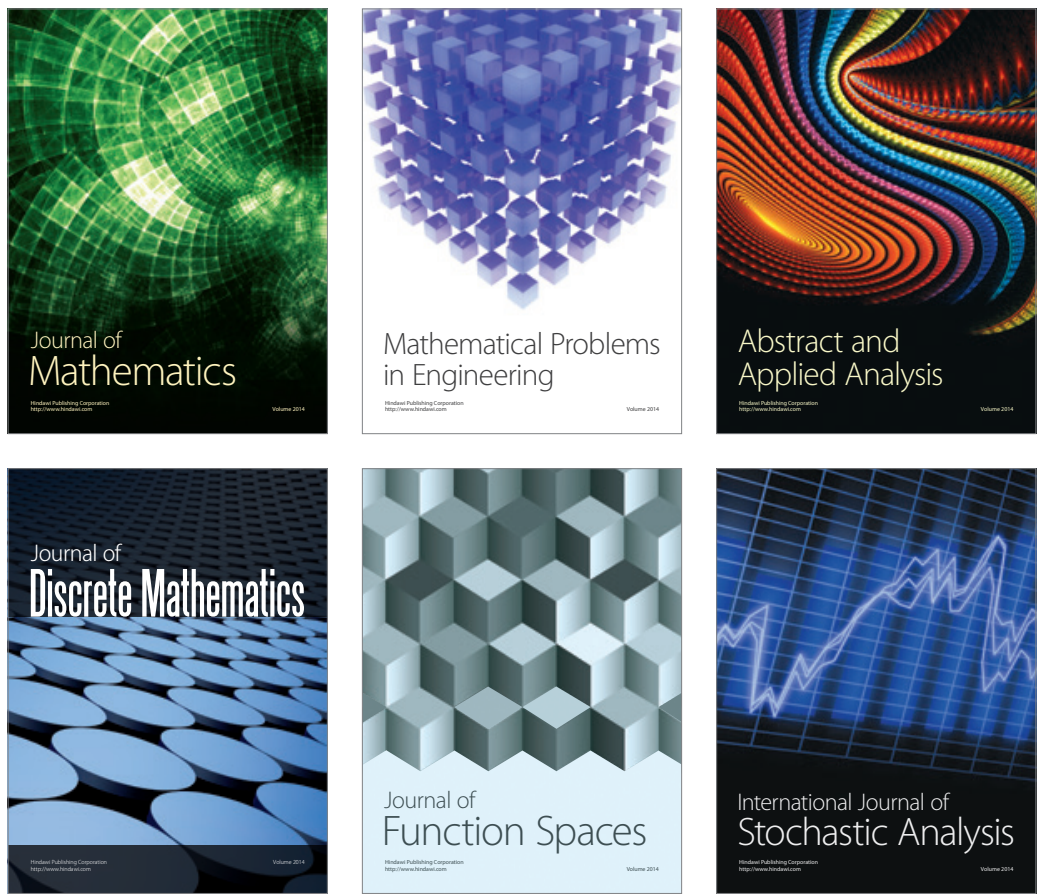

Journal of

Function Spaces

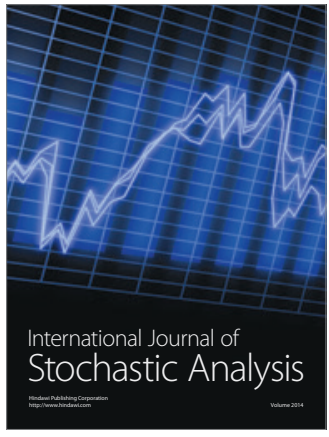

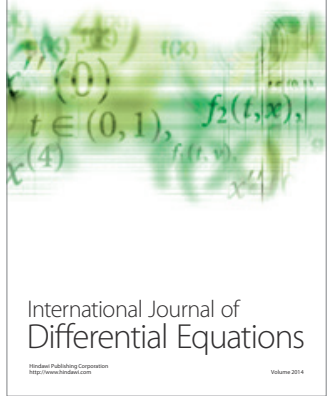
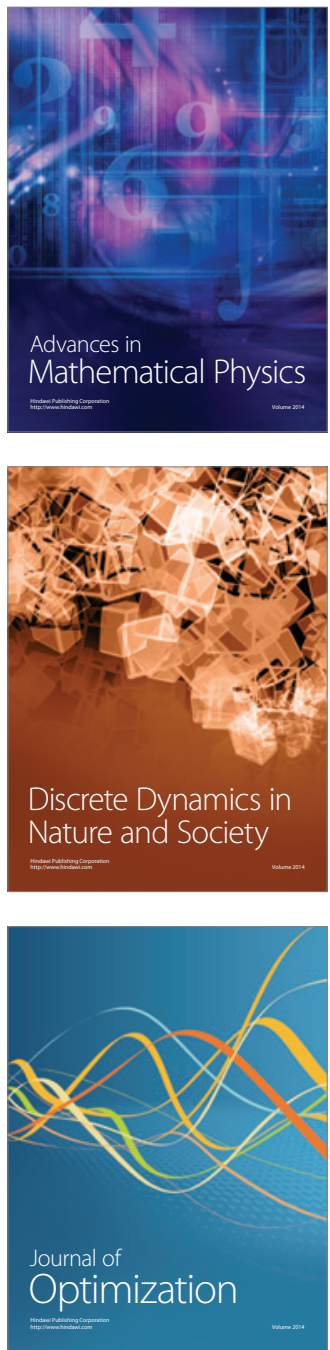